\title{
Monomial $G$-posets and their Lefschetz invariants
}

Serge Bouc ${ }^{\mathrm{a}}$, Hatice Mutlu ${ }^{\mathrm{b}, *}$

a CNRS-LAMFA, Université de Picardie - Jules Verne, 33 rue St Leu, 80039 - Amiens, France

b Department of Mathematics, Bilkent University, 06800 - Bilkent, Ankara, Turkey

\section{A R T I C L E I N F O}

\section{Article history:}

Received 15 August 2018

Available online 14 March 2019

Communicated by Markus

Linckelmann

\section{$M S C$ : \\ 06A 11 \\ 19A22 \\ $20 \mathrm{~J} 15$}

Keywords:

Burnside ring

Monomial

Tensor induction

Lefschetz invariant
A B S T R A C T

Let $G$ be a finite group, and $C$ be an abelian group. We introduce the notions of $C$-monomial $G$-sets and $C$-monomial $G$-posets, and state some of their categorical properties. This gives in particular a new description of the $C$-monomial Burnside ring $B_{C}(G)$. We also introduce Lefschetz invariants of $C$-monomial $G$-posets, which are elements of $B_{C}(G)$. These invariants allow for a definition of a generalized tensor induction multiplicative map $\mathcal{T}_{U, \lambda}: B_{C}(G) \rightarrow B_{C}(H)$ associated to any $C$-monomial $(G, H)$-biset $(U, \lambda)$, which in turn gives a group homomorphism $B_{C}(G)^{\times} \rightarrow B_{C}(H)^{\times}$ between the unit groups of $C$-monomial Burnside rings.

(C) 2019 Elsevier Inc. All rights reserved.

\section{Introduction}

Let $G$ be a finite group, and $C$ be an abelian group. In this work, we first introduce the notion of $C$-monomial $G$-set: this is a pair $(X, \mathfrak{l})$ consisting of a finite $G$-set $X$, together with a functor from the transporter category $\widehat{X}$ of $X$, to the groupoid $\bullet_{C}$ with one object and automorphism group $C$. The $C$-monomial $G$-sets form a category ${ }_{C} M G$-set, and we show that it is equivalent to the category ${ }_{C} F G$-set of $C$-fibred $G$-sets considered by

\footnotetext{
* Corresponding author.

E-mail addresses: serge.bouc@u-picardie.fr (S. Bouc), hatice.mutlu@bilkent.edu.tr (H. Mutlu).
} 
Barker ([1]). In particular, the $C$-monomial Burnside ring $B_{C}(G)$ introduced by Dress ([5]) is isomorphic to the Grothendieck ring of the category ${ }_{C} M G$-set.

We extend these definitions to the notion of $C$-monomial $G$-poset: this is a pair $(X, \mathfrak{l})$ consisting of a finite $G$-poset $X$, and a functor $\mathfrak{l}$ from the transporter category $\widehat{X}$ to $\bullet C$. We associate to each such pair $(X, \mathfrak{l})$ a Lefschetz invariant $\Lambda_{(X, \mathfrak{l})}$ lying in $B_{C}(G)$. We show that any element of $B_{C}(G)$ is equal to the Lefschetz invariant of some (non unique) $C$-monomial $G$-poset.

We also introduce the category ${ }_{C} M G$-poset of $C$-monomial $G$-posets, and show that there are natural functors of induction $\operatorname{Ind}_{H}^{G}:{ }_{C} M H$-poset $\rightarrow{ }_{C} M G$-poset and of restriction $\operatorname{Res}_{H}^{G}:{ }_{C} M G$-poset $\rightarrow{ }_{C} M H$-poset, whenever $H$ is a subgroup of $G$. These functors are compatible with the construction of Lefschetz invariants.

We extend several classical properties of the Lefschetz invariants of $G$-posets to Lefschetz invariants of $C$-monomial $G$-posets (the classical case being the case where $C$ is trivial).

We next turn to the construction of generalized tensor induction functors

$$
T_{U, \lambda}:{ }_{C} M G \text {-poset } \rightarrow{ }_{C} M H \text {-poset }
$$

associated, for arbitrary finite groups $G$ and $H$, to any $C$-monomial $(G, H)$-biset $(U, \lambda)$. We show that these functors induce well defined tensor induction maps

$$
\mathcal{T}_{U, \lambda}: B_{C}(G) \rightarrow B_{C}(H)
$$

which are not additive in general, but multiplicative and preserve identity elements. In particular, we get induced group homomorphisms between the corresponding unit groups of monomial Burnside rings, similar to those obtained by Carman ([4]) for other usual representation rings.

We show moreover that under an additional assumption, these tensor induction functors and their associated tensor induction maps are well behaved for composition. This yields to a (partial) fibred biset functor structure on the group of units of the monomial Burnside ring.

\section{The monomial Burnside ring}

Let $G$ be a finite group and $C$ be an abelian group which is noted multiplicatively. We denote by $G$-set the category of finite $G$-sets (with $G$-equivariant maps as morphisms), and $B(G)$ the usual Burnside ring of $G$, i.e. the Grothendieck ring of $G$-set for relations given by disjoint union decompositions of finite $G$-sets.

\subsection{The category of $C$-fibred $G$-sets}

A $C$-fibred $G$-set is defined to be a $C$-free $(C \times G)$-set with finitely many $C$-orbits. Let ${ }_{C} F G$-set denote the category of $C$-fibred $G$-sets where morphisms are $(C \times G)$-equivariant 
maps. The coproduct of $C$-fibred $G$-sets $X, Y$ is their coproduct $X \sqcup Y$ as sets, with the obvious $(C \times G)$-action. If $X$ and $Y$ are $C$-fibred $G$-sets, there is a $C$-action on $X \times Y$ defined by $c(x, y)=\left(c x, c^{-1} y\right)$ for any $c \in C$ and $(x, y) \in X \times Y$. The $C$-orbit of an element $(x, y)$ of $X \times Y$ is denoted by $x \otimes y$ and the set of $C$-orbits is denoted by $X \otimes Y$. Moreover $C \times G$ acts on $X \otimes Y$ by

$$
(c, g)(x \otimes y)=c g x \otimes g y
$$

for any $(c, g) \in C \times G$ and $x \otimes y \in X \otimes Y$. One checks easily that $X \otimes Y$ is again a $C$-fibred $G$-set, called the tensor product of $X$ and $Y$.

We denote the isomorphism class of a $C$-fibred $G$-set $X$ by $[X]$. The $C$-monomial Burnside ring $B_{C}(G)$, introduced by Dress ([5]), is defined as the Grothendieck group of the category of $C$-fibred $G$-sets, for relations given by $[X]+[Y]=[X \sqcup Y]$. The ring structure of $B_{C}(G)$ is induced by $[X] \cdot[Y]=[X \otimes Y]$. The identity element is the set $C$ with trivial $G$-action and the zero element is the empty set. If $C$ is trivial we recover the ordinary Burnside ring of the group $G$.

Given a $C$-fibred $G$-set $X$, we denote the set of $C$-orbits on $X$ by $C \backslash X$. The group $G$ acts on $C \backslash X$, and $X$ is $(C \times G)$-transitive if and only if $C \backslash X$ is $G$-transitive. If $C \backslash X$ is transitive as a $G$-set it is isomorphic to $G / U$ for some $U \leq G$. There exists a group homomorphism $\mu: U \rightarrow C$ such that if $U$ is the stabilizer of the orbit $C x$, then $a x=$ $\mu(a) x$ for all $a \in U$. Since the stabilizer $(C \times G)_{x}$ of $x$ in $C \times G$ is equal to

$$
(C \times G)_{x}=\left\{\left(\mu(a)^{-1}, a\right) \mid a \in U\right\}
$$

the $C$-fibred $G$-set $X$ is determined up to isomorphism by the subgroup $U$ and $\mu$.

Conversely, let $U$ be a subgroup of $G$, and $\mu: U \rightarrow C$ be a group homomorphism. Then we set $U_{\mu}=\left\{\left(\mu(a)^{-1}, a\right) \mid a \in U\right\}$, and denote by $[U, \mu]_{G}$ the $C$-fibred $G$-set $(C \times G) / U_{\mu}$. The pair $(U, \mu)$ is called a $C$-subcharacter of $G$. We denote the set of $C$-subcharacters by $\operatorname{ch}(G)$. The group $G$ acts on $\operatorname{ch}(G)$ by conjugation. The $G$-set $\operatorname{ch}(G)$ is a poset with the relation $\leq$ defined by

$$
(U, \mu) \leq(V, \nu) \Leftrightarrow U \leq V \text { and } \operatorname{Res}_{U}^{V} \nu=\mu
$$

for any $(U, \mu)$ and $(V, \nu)$ in $\operatorname{ch}(G)$.

As an abelian group we have

$$
B_{C}(G)=\bigoplus_{(U, \mu) \in G} \mathbb{Z}[U, \mu]_{G}
$$

where $(V, \nu)$ runs over $G$-representatives of the $C$-subcharacters of $G$, details can be seen in [1]. 


\subsection{The category of $C$-monomial $G$-sets}

Let $G$ be a finite group and $C$ be an abelian group. Given a $G$-set $X$, we consider its transporter category $\widehat{X}$ whose objects are the elements of $X$ and given $x, y$ in $X$ the set of morphisms from $x$ to $y$ is

$$
\operatorname{Hom}_{\widehat{X}}(x, y)=\{g \in G \mid g x=y\} .
$$

Let $\bullet_{C}$ denote the category with one object where morphisms are the elements of $C$ and composition is multiplication in $C$. Now we define $C$-monomial $G$-sets as follows.

Definition 1. A $C$-monomial $G$-set is a pair $(X, \mathfrak{l})$ consisting of a finite $G$-set $X$ and a functor $\mathfrak{l}: \widehat{X} \rightarrow \bullet_{C}$.

In other words, for each $x, y \in X$ and $g \in G$ such that $g x=y$, we have an element $\mathfrak{l}(g, x, y)$ of $C$, with the property that $\mathfrak{l}(h, y, z) \mathfrak{l}(g, x, y)=\mathfrak{l}(h g, x, z)$ if $h \in G$ and $h y=z$, and $\mathfrak{l}(1, x, x)=1$ for any $x \in X$.

Let $(X, \mathfrak{l})$ and $(Y, \mathfrak{m})$ be $C$-monomial $G$-sets. If $f: X \rightarrow Y$ is a map of $G$-sets, we slightly abuse notation and also denote by $f: \widehat{X} \rightarrow \widehat{Y}$ the obvious functor induced by $f$. Now a map $(f, \lambda):(X, \mathfrak{l}) \rightarrow(Y, \mathfrak{m})$ of $C$-monomial $G$-sets is a pair consisting of a map $f: X \rightarrow Y$ of $G$-sets and a natural transformation $\lambda: \mathfrak{l} \rightarrow \mathfrak{m} \circ f$. We denote by $C M G$-set the category whose objects are $C$-monomial $G$-sets, morphisms are the maps of $C$-monomial $G$-sets, and composition is the obvious one.

Let $(X, \mathfrak{l})$ and $\left(X^{\prime}, \mathfrak{l}^{\prime}\right)$ be $C$-monomial $G$-sets. We define the disjoint union of $C$-monomial $G$-sets as $(X, \mathfrak{l}) \sqcup\left(X^{\prime}, \mathfrak{l}^{\prime}\right)=\left(X \sqcup X^{\prime}, \mathfrak{l} \sqcup \mathfrak{l}^{\prime}\right)$ where $X \sqcup X^{\prime}$ is the disjoint union of $G$-sets and

$$
\mathfrak{l} \sqcup \mathfrak{l}^{\prime}: \widehat{X \sqcup X^{\prime}} \rightarrow \bullet_{C}
$$

is the functor such that

$$
\left(\mathfrak{l} \sqcup \mathfrak{l}^{\prime}\right)\left(g, z_{1}, z_{2}\right)= \begin{cases}\mathfrak{l}\left(g, z_{1}, z_{2}\right) & z_{1}, z_{2} \in X \\ \mathfrak{l}^{\prime}\left(g, z_{1}, z_{2}\right) & z_{1}, z_{2} \in X^{\prime}\end{cases}
$$

for any $z_{1}, z_{2} \in X \sqcup X^{\prime}$ such that $g z_{1}=z_{2}$ for some $g \in G$.

The product of $C$-monomial $G$-sets $(X, \mathfrak{l}),\left(X^{\prime}, \mathfrak{l}^{\prime}\right)$ is defined to be $\left(X \times X^{\prime}, \mathfrak{l} \times \mathfrak{l}^{\prime}\right)$ where $X \times X^{\prime}$ is the product of $G$-sets and $\mathfrak{l} \times \mathfrak{l}^{\prime}: \widehat{X \times Y} \rightarrow \bullet_{C}$ is the functor defined by

$$
\left(\mathfrak{l} \times \mathfrak{l}^{\prime}\right)\left(g,\left(x, x^{\prime}\right),\left(y, y^{\prime}\right)\right)=\mathfrak{l}(g, x, y) \mathfrak{l}^{\prime}\left(g, x^{\prime}, y^{\prime}\right)
$$

for $g \in G$ and $\left(x, x^{\prime}\right),\left(y, y^{\prime}\right) \in X \times X^{\prime}$ such that $g\left(x, x^{\prime}\right)=\left(y, y^{\prime}\right)$.

Our goal is to show that the categories ${ }_{C} M G$-set and ${ }_{C} F G$-set are equivalent. For this, we define a functor $F:{ }_{C} M G$-set $\rightarrow{ }_{C} F G$-set as follows: given a $C$-monomial $G$-set $(X, \mathfrak{l})$, we set 


$$
F(X, \mathfrak{l})=C \times \mathfrak{l} X
$$

which is the direct product $C \times X$ endowed with the $(C \times G)$-action defined by $(k, g)(c, x)=(k c l(g, x, g x), g x)$ for any $(k, g) \in C \times G$ and $(c, x) \in C \times X$.

Given a map $(f, \lambda):(X, \mathfrak{l}) \rightarrow(Y, \mathfrak{m})$ of $C$-monomial $G$-sets, we define

$$
F(f, \lambda): C \times_{\mathfrak{l}} X \rightarrow C \times_{\mathfrak{m}} Y
$$

by $F(f, \lambda)(c, x)=\left(c \lambda_{x}, f(x)\right)$ for any $(c, x) \in C \times_{\mathfrak{l}} X$. Then $F(f, \lambda)$ is a $(C \times G)$-map: indeed, given $(k, g) \in C \times G$ and $(c, x) \in C \times X$, we have

$$
\begin{aligned}
(k, g) F(f, \lambda)(c, x) & =(k, g)\left(c \lambda_{x}, f(x)\right)=\left(k c \lambda_{x} \mathfrak{m}(g, f(x), f(g x)), f(g x)\right) \\
& =\left(k c \lambda_{g x} \mathfrak{l}(g, x, g x), f(g x)\right)=F(f, \lambda)(k c \mathfrak{l}(g, x, g x), g x) \\
& =F(f, \lambda)((k, g)(c, x)) .
\end{aligned}
$$

It is clear that $F:{ }_{C} M G$-set $\rightarrow{ }_{C} F G$-set is a functor.

Lemma 2. Let $C$ be an abelian group and $G$ be a finite group. Then the above functor $F:{ }_{C} M G$-set $\rightarrow{ }_{C} F G$-set is an equivalence of categories.

Proof. We prove that $F$ is fully faithful and essentially surjective. First we show that $F$ is essentially surjective. Given a $C$-fibred $G$-set $X$, let $C \backslash X$ be the set of $C$-orbits. Clearly $C \backslash X$ is a $G$-set. We define a functor $\mathfrak{l}: \widehat{C \backslash X} \rightarrow \bullet_{C}$. Let $C x, C y \in C \backslash X$ such that $C g x=C y$ for some $g \in G$. Then there exists a unique $c \in C$ such that $g x=c y$. We set $\mathfrak{l}(g, C x, C y)=c$. We have $F(C \backslash X, \mathfrak{l})=C \times_{\mathfrak{l}}(C \backslash X)$. Now choose a set $[C \backslash X]$ of $G$-representatives of the $G$-action on $C \backslash X$. Then for any $x \in X$, there exists a unique $C \sigma_{x} \in[C \backslash X]$ such that $x \in C \sigma_{x}$. Since $X$ is $C$-free, there exists a unique $c_{x} \in C$ such that $x=c_{x} \sigma_{x}$. We define a $(C \times G)$-map $f: X \rightarrow C \times \times_{\mathfrak{l}}(C \backslash X)$ such that $f(x)=\left(c_{x}, C \sigma_{x}\right)$. Then

$$
\begin{aligned}
(c, g) f(x) & =(c, g)\left(c_{x}, C \sigma_{x}\right)=\left(c_{x} c l\left(g, C \sigma_{x}, C g \sigma_{x}\right), C g \sigma_{x}\right)=\left(c_{x} c, C g \sigma_{x}\right) \\
& =\left(c_{c g x}, C g \sigma_{x}\right)=f((c, g) x) .
\end{aligned}
$$

So $f$ is a $(C \times G)$-map and clearly an isomorphism. Thus, $F$ is essentially surjective.

Let $(X, \mathfrak{l})$ and $(Y, \mathfrak{m})$ be $C$-monomial $G$-sets. We need to show that the map

$$
\bar{F}: \operatorname{Hom}((X, \mathfrak{l}),(Y, \mathfrak{m})) \rightarrow \operatorname{Hom}(F(X, \mathfrak{l}), F(Y, \mathfrak{m}))
$$

induced by $F$ is surjective and injective. Let $\varphi: C \times_{\mathfrak{l}} X \rightarrow C \times_{\mathfrak{m}} Y$ be a $(C \times G)$-map. Given $(1, x) \in C \times{ }_{\mathfrak{l}} X$, let $\varphi(1, x)=\left(c_{x}, z_{x}\right)$ for $\left(c_{x}, z_{x}\right) \in C \times Y$. Since $\varphi$ is a $(C \times G)$-map, we get 


$$
\varphi(c, x)=\left(c c_{x}, z_{x}\right)
$$

and

$$
\varphi(1, g x)=\left(c_{x} \mathfrak{m}\left(g, z_{x}, g z_{x}\right) \mathfrak{l}^{-1}(g, x, g x), g z_{x}\right)
$$

for any $c \in C$ and $g \in G$. We define a map

$$
(f, \lambda):(X, \mathfrak{l}) \rightarrow(Y, \mathfrak{m})
$$

such that $f: X \rightarrow Y$ is defined by $f(x)=z_{x}$ and $\lambda: \mathfrak{l} \rightarrow \mathfrak{m} \circ f$ is defined by $\lambda_{x}=c_{x}$ for any $x \in X$. Clearly, $f$ is a $G$-set map. Let $x \in X$ and $g \in G$. Then

$$
\begin{aligned}
\mathfrak{m}(g, f(x), f(g x)) \lambda_{x} & =\mathfrak{m}(g, f(x), f(g x)) c_{x}=c_{x} \mathfrak{m}(g, f(x), f(g x)) \mathfrak{l}^{-1}(g, x, g x) \mathfrak{l}(g, x, g x) \\
& =\mathfrak{l}(g, x, g x) c_{g x}=\mathfrak{l}(g, x, g x) \lambda_{g x} .
\end{aligned}
$$

So $\lambda: \mathfrak{l} \rightarrow \mathfrak{m} \circ f$ is a natural transformation and $(f, \lambda)$ is a map of $C$-monomial $G$-sets. Thus, $\bar{F}(f, \lambda)=\varphi$ and $\bar{F}$ is surjective. The injectivity is clear, so $F$ is fully faithful.

Proposition 3. Let $G$ be a finite group. Then $B_{C}(G)$ is isomorphic to the Grothendieck ring of the category ${ }_{C} M G$-set, for relations given by decomposition into disjoint unions of $C$-monomial $G$-sets and multiplication induced by product of $C$-monomial $G$-sets.

Proof. We let $B_{C}^{1}(G)$ denote the Grothendieck ring of the category ${ }_{C} M G$-set. The equivalence

$$
F:{ }_{C} M G \text {-set } \rightarrow{ }_{C} F G \text {-set }
$$

induces a bijection

$$
\widehat{F}: B_{C}^{1}(G) \rightarrow B_{C}(G)
$$

such that

$$
\widehat{F}([(X, \mathfrak{l})])=[C \times \mathfrak{l} X]
$$

for any $C$-monomial $G$-set $(X, \mathfrak{l})$. Now we show that $\widehat{F}$ is a ring homomorphism. Let $\left(X_{1}, \mathfrak{l}_{1}\right)$ and $\left(X_{2}, \mathfrak{l}_{2}\right)$ be $C$-monomial $G$-sets. Then

$$
\begin{gathered}
\widehat{F}\left(\left[\left(X_{1}, \mathfrak{l}_{1}\right)\right]+\left[\left(X_{1}, \mathfrak{l}_{1}\right)\right]\right)=\widehat{F}\left(\left[\left(X_{1}, \mathfrak{l}_{1}\right) \sqcup\left(X_{1}, \mathfrak{l}_{1}\right)\right]\right)=\widehat{F}\left(\left[\left(X_{1} \sqcup X_{2}, \mathfrak{l}_{1} \sqcup \mathfrak{l}_{2}\right)\right]\right) \\
\quad=\left[C \times_{\mathfrak{l}_{1} \sqcup \mathfrak{l}_{2}}\left(X_{1} \sqcup X_{2}\right)\right]=\left[\left(X_{1}, \mathfrak{l}_{1}\right) \sqcup\left(X_{2}, \mathfrak{l}_{2}\right)\right]=\left[C \times_{\mathfrak{l}_{1}} X_{1}\right]+\left[C \times_{\mathfrak{l}_{2}} X_{2}\right] .
\end{gathered}
$$


For multiplicativity of $\widehat{F}$ we define a map

$$
f: C \times \mathfrak{l}_{1} \times \mathfrak{l}_{2}\left(X_{1} \times X_{2}\right) \rightarrow\left(C \times \mathfrak{l}_{1} X_{1}\right) \times{ }_{C}\left(C \times \mathfrak{l}_{2} X_{2}\right)
$$

such that $f\left(c,\left(x_{1}, x_{2}\right)\right)=\left(c, x_{1}\right) \times_{C}\left(1, x_{2}\right)$. Let $(k, g) \in C \times G$ and $\left(c,\left(x_{1}, x_{2}\right)\right) \in$ $C \times \mathfrak{l}_{1} \times \mathfrak{l}_{2}\left(X_{1} \times X_{2}\right)$. Then

$$
\begin{aligned}
(k, g) f\left(c,\left(x_{1}, x_{2}\right)\right) & =(k, g)\left(\left(c, x_{1}\right) \times_{C}\left(1, x_{2}\right)\right)=\left((k, g)\left(c, x_{1}\right) \times_{C}(1, g)\left(1, x_{2}\right)\right) \\
& =\left(k c l_{1}\left(g, x_{1}, g x_{1}\right), g x_{1}\right) \times_{C}\left(\mathfrak{l}_{2}\left(g, x_{2}, g x_{2}\right), g x_{2}\right) \\
& =\left(k c l_{1}\left(g, x_{1}, g x_{1}\right) \mathfrak{l}_{2}\left(g, x_{2}, g x_{2}\right), g x_{1}\right) \times_{C}\left(1, g x_{2}\right) \\
& =f\left(k c l_{1}\left(g, x_{1}, g x_{1}\right) \mathfrak{l}_{2}\left(g, x_{2}, g x_{2}\right), g\left(x_{1}, x_{2}\right)\right) \\
& =f\left((k, g)\left(c,\left(x_{1}, x_{2}\right)\right)\right) .
\end{aligned}
$$

So $f$ is a $(C \times G)$-map and obviously, $f$ is a $(C \times G)$-isomorphism. Using $f$ we get

$$
\begin{aligned}
\widehat{F}\left(\left[X_{1}, \mathfrak{l}_{1}\right] \cdot\left[X_{2}, \mathfrak{l}_{2}\right]\right) & =\widehat{F}\left(\left[X_{1} \times X_{2}, \mathfrak{l}_{1} \times \mathfrak{l}_{2}\right]\right)=\left[C \times \mathfrak{l}_{2} \times \mathfrak{l}_{2}\left(X_{1} \times X_{2}\right)\right] \\
& =\left[\left(C \times \mathfrak{l}_{1} X_{1}\right) \times_{C}\left(C \times_{\mathfrak{l}_{2}} X_{2}\right)\right] .
\end{aligned}
$$

Thus, the desired result follows.

Remark 4. Let $(X, \mathfrak{l})$ be a $C$-monomial $G$-set. For all $x \in X$, we get a character $\mathfrak{l}_{x}$ : $G_{x} \rightarrow C$ defined by $\mathfrak{l}_{x}(g)=\mathfrak{l}(g, x, x)$ for $g \in G_{x}$. On the other hand given a subgroup $U$ of $G$ and a group homomorphism $\mu: U \rightarrow C$ we get a $C$-monomial $G$-set $(G / U, \widehat{\mu})$ where and $\widehat{\mu}: \widehat{G / U} \rightarrow \bullet_{C}$ is the functor such that given $g U, k U \in G / U$ if $h g U=k U$ for some $g \in G$ then $\widehat{\mu}(h, g U, k U)=\mu\left(k^{-1} h g\right)$. Moreover, $[U, \mu]_{G}$ and $[G / U, \widehat{\mu}]$ represents the same element in $B_{C}(G)$.

\subsection{The Lefschetz invariant attached to a monomial G-poset}

A $G$-poset $X$ is a partially ordered set $(X, \leq)$ with a compatible $G$-action (that is $g x \leq g y$ whenever $g \in G$ and $x \leq y$ in $X$ ). A map of $G$-posets is a $G$-equivariant map of posets. We denote by $G$-poset the category of finite $G$-posets obtained in this way.

There is an obvious functor $\iota_{G}: G$-set $\rightarrow G$-poset sending each finite $G$-set to the set $X$ ordered by the equality relation, and each $G$-equivariant map to itself.

The Lefschetz invariant attached to a finite $G$-poset, which is an element of the Burnside ring of $G$ has been introduced in [7] by Thévenaz. We will define similarly a Lefschetz invariant attached to a $C$-monomial $G$-poset as an element of the $C$-monomial Burnside ring of $G$. 


\subsubsection{The category of $C$-monomial $G$-posets}

Given a $G$-poset $X$, we consider the category $\widehat{X}$ whose objects are the elements of $X$ and given $x, y$ in $X$ the set of morphisms from $x$ to $y$ is

$$
\operatorname{Hom}_{\widehat{X}}(x, y)=\{g \in G \mid g x \leq y\}
$$

Now we define a $C$-monomial $G$-poset as follows.

Definition 5. A $C$-monomial $G$-poset is a pair $(X, \mathfrak{l})$ consisting of a $G$-poset $X$ and a functor $\mathfrak{l}: \widehat{X} \rightarrow \bullet_{C}$.

In other words, for each $x, y \in X$ and $g \in G$ such that $g x \leq y$, we have an element $\mathfrak{l}(g, x, y)$ of $C$, with the property that $\mathfrak{l}(h, y, z) \mathfrak{l}(g, x, y)=\mathfrak{l}(h g, x, z)$ if $h \in G$ and $h y \leq z$, and $\mathfrak{l}(1, x, x)=1$ for any $x \in X$.

Let $(X, \mathfrak{l})$ and $(Y, \mathfrak{m})$ be $C$-monomial $G$-posets. $A$ map of $C$-monomial $G$-posets from $(X, \mathfrak{l})$ to $(Y, \mathfrak{m})$ is a pair $(f, \lambda):(X, \mathfrak{l}) \rightarrow(Y, \mathfrak{m})$, where $f: X \rightarrow Y$ is a map of $G$-posets and $\lambda: \mathfrak{l} \rightarrow \mathfrak{m} \circ f$ is a natural transformation. We denote the category of $C$-monomial $G$-posets by ${ }_{C} M G$-poset. Product and disjoint union of $C$-monomial $G$-posets are defined as for $C$-monomial $G$-sets. When $C$ is the trivial group, we will identify the category ${ }_{C} M G$-poset with $G$-poset.

Remark 6. If $(X, \mathfrak{l})$ is a $C$-monomial $G$-poset, then for any $x \in X$ we get a character $\mathfrak{l}_{x}: G_{x} \rightarrow C$ defined by $\mathfrak{l}_{x}(g)=\mathfrak{l}(g, x, x)$. Moreover, if $x \leq y$, then

$$
\operatorname{res}_{G_{x} \cap G_{y}}^{G_{x}} \mathfrak{l}_{x}=\operatorname{res}_{G_{x} \cap G_{y}}^{G_{y}} \mathfrak{l}_{y}
$$

because we have the following commutative diagram:

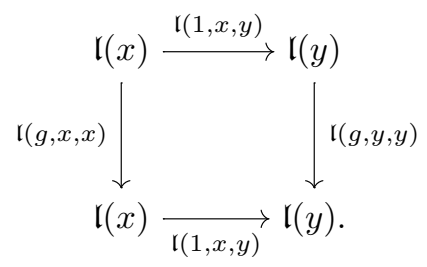

Let $H$ be a subgroup of $G$ and $(X, \mathfrak{l})$ be a $C$-monomial $H$-set. We let $G \times_{H} X$ to be the quotient of $G \times X$ by the action of $H$. The set $G \times_{H} X$ is a $G$-set via the action $g\left(u,{ }_{H} x\right)=\left(g u,{ }_{H} x\right)$, for any $g \in G$, and $\left(u,{ }_{H} x\right) \in G \times{ }_{H} X$. We define an order relation $\leq$ on $G \times_{H} X$ as

$$
\forall\left(u,{ }_{H} x\right),\left(v,_{H} y\right) \in G \times_{H} X,\left(u,{ }_{H} x\right) \leq\left(v,_{H} y\right) \Leftrightarrow \exists h \in H, u=v h, x \leq h^{-1} y .
$$




$$
G \times{ }_{H} X=\bigsqcup_{g \in G / H} g \times_{H} X
$$

it's enough to consider the chains of type $\left(u,{ }_{H} x_{0}\right)<\ldots<\left(u,{ }_{H} x_{n}\right)$ in $G \times_{H} X$ for some $u \in G$ and a chain $x_{0}<\ldots<x_{n}$ in $X$ for some $n \in \mathbb{N}$.

Let $\left(u,{ }_{H} x\right),\left(u,{ }_{H} y\right) \in G \times{ }_{H} X$ and $g \in G$ such that $g\left(u,{ }_{H} x\right) \leq\left(u,{ }_{H} y\right)$. Then there exists $h \in H$ such that $g u=u h$ and $h x \leq y$. We define the induced $C$-monomial $G$-poset $\operatorname{Ind}_{H}^{G}(X, \mathfrak{l})$ of $(X, \mathfrak{l})$ as the pair $\left(G \times_{H} X, G \times_{H} \mathfrak{l}\right)$ where $G \times_{H} \mathfrak{l}: \widehat{G \times{ }_{H}} X \rightarrow \bullet_{C}$ is defined by

$$
\left(G \times{ }_{H} \mathfrak{l}\right)\left(g,\left(u,{ }_{H} x\right),\left(u,{ }_{H} y\right)\right)=\mathfrak{l}(h, x, y) .
$$

Now show that $\left(G \times_{H} X, G \times_{H} \mathfrak{l}\right)$ is a $C$-monomial $G$-poset.

Let $\left(u,{ }_{H} x\right),\left(u,{ }_{H} y\right),\left(u,{ }_{H} z\right) \in G \times{ }_{H} X$ such that

$$
g\left(u,{ }_{H} x\right) \leq\left(u,{ }_{H} y\right)
$$

and

$$
g^{\prime}\left(u,{ }_{H} y\right) \leq\left(u,{ }_{H} z\right)
$$

for some $g, g^{\prime} \in G$. Then there exist some $h, h^{\prime} \in H$ such that

$$
g u=u h, \quad g^{\prime} u=u h^{\prime}, \quad h x \leq y, h^{\prime} y \leq z
$$

Then $t=h^{\prime} h \in H$. Moreover $g^{\prime} g u=u h^{\prime} h=u t$ and $t x=h^{\prime} h x \leq z$. Now we get

$$
\begin{aligned}
& \left(G \times{ }_{H} \mathfrak{l}\right)\left(g^{\prime} g,\left(u,{ }_{H} x\right),\left(u,{ }_{H} z\right)\right)=\mathfrak{l}(t, x, z)=\mathfrak{l}\left(h^{\prime} h, x, z\right)=\mathfrak{l}\left(h^{\prime}, y, z\right) \mathfrak{l}(h, x, y) \\
& \quad=\left(G \times{ }_{H} \mathfrak{l}\right)\left(g^{\prime},\left(u,{ }_{H} x\right),\left(u,{ }_{H} y\right)\right)\left(G \times{ }_{H} \mathfrak{l}\right)\left(g,\left(u,{ }_{H} y\right),\left(u,{ }_{H} z\right)\right) .
\end{aligned}
$$

We also have $\left(G \times_{H} \mathfrak{l}\right)\left(1,\left(u,,_{H} x\right),\left(u,{ }_{H} x\right)\right)=1$ for any $\left(u,{ }_{H} x\right) \in G \times_{H} X$. Thus $G \times_{H} \mathfrak{l}$ is a functor. So $\operatorname{Ind}_{H}^{G}(X, \mathfrak{l})$ is a $C$-monomial $G$-poset.

Given a $C$-monomial $G$-poset $(Y, \mathfrak{m})$, the restriction $\operatorname{Res}_{H}^{G}(Y, \mathfrak{m})$ of $(Y, \mathfrak{m})$ is the pair ( $\operatorname{Res}_{H}^{G} Y, \operatorname{res}_{H}^{G} \mathfrak{m}$ ) where $\operatorname{Res}_{H}^{G} Y$ is the restriction of the $G$-poset $Y$ to $H$-poset and $\operatorname{res}_{H}^{G} \mathfrak{m}$ is the restriction of the functor $\mathfrak{m}$ from $\widehat{Y}$ to $\widehat{\operatorname{Res}_{H}^{G} Y}$.

Proposition 7. Let $G$ be a finite group.

1. If $Y$ is a finite $G$-poset, denote by $1_{Y}: \widehat{Y} \rightarrow \bullet_{C}$ the trivial functor defined by $1_{Y}(g, x, y)=1$ for any $g \in G$ and $x, y \in Y$ such that $g x \leq y$. Then the assignment $Y \mapsto\left(Y, 1_{Y}\right)$ is a functor $\tau_{G}$ from $G$-poset to ${ }_{C} M G$-poset.

2. Let $H$ be a subgroup of $G$. The assignment $(X, \mathfrak{l}) \mapsto \operatorname{Ind}_{H}^{G}(X, \mathfrak{l})$ is a functor $\operatorname{Ind}_{H}^{G}$ : ${ }_{C} M H$-poset $\rightarrow{ }_{C} M G$-poset, and the assignment $(Y, \mathfrak{m}) \mapsto \operatorname{Res}_{H}^{G}(Y, \mathfrak{m})$ is a functor $\operatorname{Res}_{H}^{G}:{ }_{C} M G$-poset $\rightarrow{ }_{C} M H$-poset. 
3. Moreover the diagrams

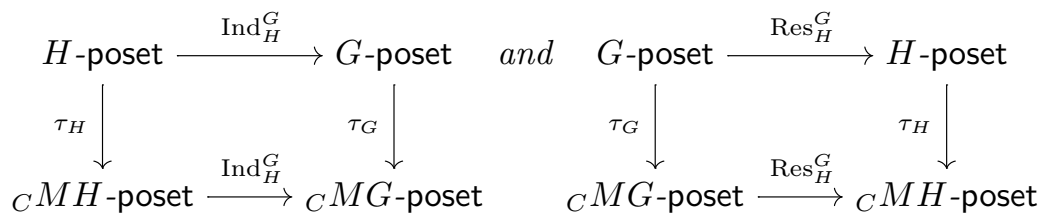

of categories and functors are commutative.

Proof. 1. Let $f: X \rightarrow Y$ be a map of $G$-posets. We set

$$
\tau_{G}(f)=\left(f, 1_{f}\right):\left(X, 1_{X}\right) \rightarrow\left(Y, 1_{Y}\right)
$$

where $1_{f}: 1_{X} \rightarrow 1_{Y} \circ f$ is defined by $1_{f_{x}}=1$ for any $x \in X$. Obviously $\left(f, 1_{f}\right)$ is a map of $C$-monomial $G$-posets and $\tau_{G}$ is a functor.

2. Let $(f, \lambda):(X, \mathfrak{l}) \rightarrow(Y, \mathfrak{m})$ be a map of $C$-monomial $H$-posets. We set the pair

$$
\operatorname{Ind}_{H}^{G}(f, \lambda)=\left(G \times_{H} f, G \times_{H} \lambda\right):\left(G \times_{H} X, G \times_{H} \mathfrak{l}\right) \rightarrow\left(G \times_{H} Y, G \times_{H} \mathfrak{m}\right)
$$

where

$$
G \times_{H} f: G \times_{H} X \rightarrow G \times_{H} Y
$$

is defined by $\left(G \times{ }_{H} f\right)\left(u,{ }_{H} x\right)=\left(u,{ }_{H} f(x)\right)$ and

$$
G \times_{H} \lambda: G \times_{H} \mathfrak{l} \rightarrow\left(G \times_{H} \mathfrak{m}\right) \circ\left(G \times_{H} f\right)
$$

is defined by $\left(G \times_{H} \lambda\right)_{\left(u,{ }_{H} x\right)}=\lambda_{x}$ for any $\left(u,{ }_{H} x\right) \in G \times_{H} X$. It's clear that $G \times_{H} f$ is a map of $C$-monomial $G$-posets. Now we show that $G \times_{H} \lambda$ is a natural transformation. Let $\left(u,{ }_{H} x\right),\left(u,{ }_{H} y\right) \in G \times{ }_{H} X$ such that $g\left(u,{ }_{H} x\right) \leq\left(u,{ }_{H} y\right)$ for some $g \in G$. Then $g u=u h$ and $h x \leq y$ for some $h \in H$. Since $\lambda: \mathfrak{l} \rightarrow \mathfrak{m} \circ f$ is a natural transformation, we get

$$
\begin{aligned}
& \left(G \times_{H} \mathfrak{m}\right)\left(g,\left(u,{ }_{H} f(x)\right),\left(u,{ }_{H} f(y)\right)\right)\left(G \times_{H} \lambda\right)_{\left(u,{ }_{H} x\right)}=\mathfrak{m}(h, f(x), f(y)) \lambda_{x} \\
& =\lambda_{y} \mathfrak{l}(h, x, y)=\left(G \times \times_{H} \lambda\right)_{\left(u,,_{H} y\right)}\left(G \times_{H} \mathfrak{l}\right)\left(g,\left(u,{ }_{H} x\right),\left(u,{ }_{H} y\right)\right) .
\end{aligned}
$$

Now consider $\left(\operatorname{id}_{X}, \operatorname{id}_{\mathfrak{l}}\right):(X, \mathfrak{l}) \rightarrow(X, \mathfrak{l})$ where $\operatorname{id}_{X}: X \rightarrow X$ is the identity map on the $H$-set $X$ and $\operatorname{id}_{\mathfrak{l}}: \mathfrak{l} \rightarrow \mathfrak{l}^{\circ} \mathrm{id}_{X}$ is the identity transformation. Then we get

$$
\operatorname{Ind}_{H}^{G}\left(\operatorname{id}_{X}, \operatorname{id}_{\mathfrak{l}}\right)=\left(\operatorname{id}_{G \times_{H} X}, \operatorname{id}_{G \times_{H}} \mathfrak{l}\right)
$$

Now let $(f, \lambda):(X, \mathfrak{l}) \rightarrow(Y, \mathfrak{m})$ and $(t, \beta):(Y, \mathfrak{m}) \rightarrow(Z, \mathfrak{r})$ be the maps of $C$-monomial $H$-posets. We obviously have 


$$
\left(G \times_{H} t\right) \circ\left(G \times_{H} f\right)=G \times_{H}(t \circ f)
$$

and

$$
\left(G \times_{H} \beta\right) \circ\left(G \times_{H} \lambda\right)=G \times_{H}(\beta \circ \lambda) .
$$

Thus,

$$
\operatorname{Ind}_{H}^{G}(t, \beta) \circ \operatorname{Ind}_{H}^{G}(f, \lambda)=\operatorname{Ind}_{H}^{G}((t, \beta) \circ(f, \lambda)) .
$$

So $\operatorname{Ind}_{H}^{G}:{ }_{C} M H$-poset $\rightarrow{ }_{C} M G$-poset is a functor.

Now let $(f, \lambda):(X, \mathfrak{l}) \rightarrow(Y, \mathfrak{m})$ be a map of $C$-monomial $G$-posets. We set the pair

$$
\operatorname{Res}_{H}^{G}(f, \lambda)=\left(\left.f\right|_{H},\left.\lambda\right|_{H}\right):\left(\operatorname{Res}_{H}^{G} X, \operatorname{res}_{H}^{G} \mathfrak{l}\right) \rightarrow\left(\operatorname{Res}_{H}^{G} Y, \operatorname{res}_{H}^{G} \mathfrak{m}\right)
$$

where $\left.f\right|_{H}: \operatorname{Res}_{H}^{G} X \rightarrow \operatorname{Res}_{H}^{G} Y$ is defined as the restriction of map of $G$-posets $f$ to map of $H$-posets and $\left.\lambda\right|_{H}:\left.\operatorname{res}_{H}^{G} \mathfrak{l} \rightarrow \operatorname{res}_{H}^{G} \mathfrak{m} \circ f\right|_{H}$ is defined as the restriction of $\lambda$. Clearly, we get that $\operatorname{Res}_{H}^{G}:{ }_{C} M G$-poset $\rightarrow{ }_{C} M H$-poset is a functor.

3. Let $X$ be an $H$-poset. Commutativity of the first diagram follows from

$$
\tau_{G} \circ \operatorname{Ind}_{H}^{G}(X)=\tau_{G}\left(G \times_{H} X\right)=\left(G \times_{H} X, 1_{G \times_{H} X}\right)=\operatorname{Ind}_{H}^{G}\left(X, 1_{X}\right)=\operatorname{Ind}_{H}^{G} \circ \tau_{H}(X)
$$

Now let $Y$ be a $G$-poset. Commutativity of the second diagram follows from

$$
\begin{aligned}
\tau_{H} \circ \operatorname{Res}_{H}^{G}(Y) & =\tau_{H}\left(\operatorname{Res}_{H}^{G} Y\right)=\left(\operatorname{Res}_{H}^{G} Y, 1_{\operatorname{Res}_{H}^{G} Y}\right) \\
& =\left(\operatorname{Res}_{H}^{G} Y, \operatorname{res}_{H}^{G} 1_{Y}\right)=\operatorname{Res}_{H}^{G}\left(Y, 1_{Y}\right)=\operatorname{Res}_{H}^{G} \circ \tau_{H}(Y) .
\end{aligned}
$$

Proposition 8. Let $G$ be a finite group and $H$ be subgroup of $G$. Then the functor $\operatorname{Ind}_{H}^{G}$ : ${ }_{C} M H$-poset $\rightarrow{ }_{C} M G$-poset is left adjoint to the functor $(Y, \mathfrak{m}) \mapsto \operatorname{Res}_{H}^{G}(Y, \mathfrak{m})$.

Proof. We prove that for any $C$-monomial $H$-poset $(X, \mathfrak{l})$ and any $C$-monomial $G$-poset $(Y, \mathfrak{m})$ we have a bijection

$$
\operatorname{Hom}_{C} M G\left(\operatorname{Ind}_{H}^{G}(X, \mathfrak{l}),(Y, \mathfrak{m})\right) \cong \operatorname{Hom}_{C} M H\left((X, \mathfrak{l}), \operatorname{Res}_{H}^{G}(Y, \mathfrak{m})\right)
$$

natural in $(X, \mathfrak{l})$ and $(Y, \mathfrak{m})$.

We define

$$
\varphi: \operatorname{Hom}_{C} M G\left(\operatorname{Ind}_{H}^{G}(X, \mathfrak{l}),(Y, \mathfrak{m})\right) \rightarrow \operatorname{Hom}_{C} M H\left((X, \mathfrak{l}), \operatorname{Res}_{H}^{G}(Y, \mathfrak{m})\right)
$$

where

$$
\varphi:(f, \lambda) \mapsto(\varphi(f), \varphi(\lambda))
$$


such that

$$
\varphi(f): X \rightarrow \operatorname{Res}_{H}^{G}(Y)
$$

defined by $\varphi(f)(x)=f\left(1,{ }_{H} x\right)$ and

$$
\varphi(\lambda): \mathfrak{l} \rightarrow \operatorname{resm} \circ \varphi(f)
$$

defined by $\left.\varphi(\lambda)_{x}=\lambda_{(1, H} x\right)$ for any $x \in X$. Obviously, $\varphi(f)$ is a map of $H$-posets. We need to show that

$$
\varphi(\lambda): \mathfrak{l} \rightarrow \operatorname{resm} \circ \varphi(f)
$$

is a natural transformation. Let $x, y \in X$ such that $g x \leq y$ for some $g \in G$. Then

$$
\begin{gathered}
\mathfrak{m}(h, \varphi(f)(x), \varphi(f)(y)) \varphi(\lambda)_{x}=\mathfrak{m}\left(h, f\left(1,{ }_{H} x\right), f\left(1,{ }_{H} y\right)\right) \lambda_{\left(1,,_{H} x\right)} \\
=\lambda_{\left(1, H_{H} y\right)} \mathfrak{l}(h, x, y)=\varphi(\lambda)_{y}\left(G \times{ }_{H} \mathfrak{l}\right)\left(h,\left(1,{ }_{H} x\right),\left(1,{ }_{H} y\right)\right) .
\end{gathered}
$$

We define an inverse map to $\varphi$ as

$$
\theta: \operatorname{Hom}_{C} M H\left((X, \mathfrak{l}), \operatorname{Res}_{H}^{G}(Y, \mathfrak{m})\right) \rightarrow \operatorname{Hom}_{C} M G\left(\operatorname{Ind}_{H}^{G}(X, \mathfrak{l}),(Y, \mathfrak{m})\right)
$$

where

$$
\theta:(\psi, \beta) \mapsto(\theta(\psi), \theta(\beta))
$$

such that

$$
\theta(\psi): G \times_{H} X \rightarrow Y
$$

defined as $\theta(\psi)\left(u,{ }_{H} x\right)=u \psi(x)$ and

$$
\theta(\beta): G \times_{H} \mathfrak{l} \rightarrow \mathfrak{m} \circ \theta(\psi)
$$

defined as

$$
\left.\theta(\beta)_{(u, H} x\right)=\mathfrak{m}(u, \psi(x), u \psi(x)) \beta_{x}
$$

for any $\left(u,{ }_{H} x\right) \in G \times{ }_{H} X$. Obviously, the map $\theta(\psi)$ is a map of $G$-posets. We need to show that $\theta(\beta)$ is a natural transformation. Let $\left(u,{ }_{H} x\right),\left(u,{ }_{H} y\right) \in G \times{ }_{H} X$ such that $g\left(u,{ }_{H} x\right) \leq\left(u,{ }_{H} y\right)$ for some $g \in G$. Then there exists some $h \in H$ such that $g u=u h$ and $h x \leq y$. Now, we have 


$$
\begin{aligned}
& \mathfrak{m}\left(g, \theta(\psi)\left(u,{ }_{H} x\right), \theta(\psi)\left(u,{ }_{H} y\right)\right) \theta(\beta)_{\left(u,{ }_{H} x\right)}=\mathfrak{m}(g, u \psi(x), u \psi(y)) \mathfrak{m}(u, \psi(x), u \psi(x)) \beta_{x} \\
& \quad=\mathfrak{m}(u, \psi(y), u \psi(y)) \mathfrak{m}(h, \psi(x), \psi(y)) \beta_{x}=\mathfrak{m}(u, \psi(y), u \psi(y)) \beta_{y} \mathfrak{l}(h, x, y) \\
& \left.\quad=\theta(\beta)_{(u, H} y\right)\left(G \times_{H} \mathfrak{l}\right)\left(h,\left(u_{H} x\right),\left(u,_{H} y\right)\right)
\end{aligned}
$$

Clearly, $\varphi$ and $\theta$ are mutual inverse maps, and natural in $(X, \mathfrak{l})$ and $(Y, \mathfrak{m})$.

\subsubsection{The Lefschetz invariant attached to a $C$-monomial $G$-poset}

Let $(X, \mathfrak{l})$ be a $C$-monomial $G$-poset. The Lefschetz invariant $\Lambda_{(X, \mathfrak{l})}$ of $(X, \mathfrak{l})$ is the element of $B_{C}(G)$ defined by

$$
\Lambda_{(X, \mathfrak{l})}=\sum_{x_{0}<\ldots<x_{n} \in G X}(-1)^{n}\left[G_{x_{0}, \ldots, x_{n}}, \operatorname{Res}_{G_{x_{0}, \ldots, x_{n}}}^{G_{x_{0}}}\left(\mathfrak{l}_{x_{0}}\right)\right]_{G}
$$

where $x_{0}<\ldots<x_{n}$ runs over $G$-representatives of the chains in $X$. The group $G_{x_{0}, \ldots, x_{n}}$ is the stabilizer of the set $\left\{x_{0}, \ldots, x_{n}\right\}$, that is $G_{x_{0}, \ldots, x_{n}}=\cap_{i=0}^{n} G_{x_{i}}$. Here $\operatorname{Res}_{G_{x_{0}, \ldots, x_{n}}}^{G_{x_{0}}}\left(\mathfrak{l}_{x_{0}}\right)$ denotes the restriction of the character $\mathfrak{l}_{x_{0}}$ introduced in Remark 4. Observe that if $x_{0}<\ldots<x_{n}$ is a chain in $X$ for some $n \in \mathbb{N}$, by Remark 6 we have

$$
\operatorname{Res}_{G_{x_{0}, \ldots, x_{n}}}^{G_{x_{0}}} \mathfrak{l}_{x_{0}}=\operatorname{Res}_{G_{x_{0}, \ldots, x_{n}}}^{G_{x_{i}}} \mathfrak{l}_{x_{i}}
$$

for any $0 \leq i \leq n$.

Let $(X, \mathfrak{l})$ be a $C$-monomial $G$-poset. Given $n \in \mathbb{N}$, let $\operatorname{Sd}_{n}(X)$ denote the set of chains in $X$ with order $n+1$. Obviously, the set $\operatorname{Sd}_{n}(X)$ is a $G$-set. Then $\left(\operatorname{Sd}_{n}(X), \mathfrak{l}_{n}\right)$ is a $C$-monomial $G$-set where $\mathfrak{l}_{n}: \widehat{\operatorname{Sd}_{n}(X)} \rightarrow \bullet_{C}$ is the functor defined by

$$
\mathfrak{l}_{n}\left(g, x_{0}<\ldots<x_{n}, y_{0}<\ldots<y_{n}\right)=\mathfrak{l}\left(g, x_{0}, y_{0}\right)
$$

for any $x_{0}<\ldots<x_{n}$, and $y_{0}<\ldots<y_{n}$ in $\operatorname{Sd}_{n}(X)$ such that

$$
g\left(x_{0}<\ldots<x_{n}\right)=y_{0}<\ldots<y_{n}
$$

for some $g \in G$.

Remark 9. Given a $C$-monomial $G$-poset $(X, \mathfrak{l})$, we have the following isomorphism of monomial $G$-sets:

$$
\left(\operatorname{Sd}_{n}(X), \mathfrak{l}_{n}\right) \cong \bigsqcup_{x_{0}<\ldots<x_{n} \in \in_{G} \operatorname{Sd}_{n}(X)}\left(G / G_{x_{0}, \ldots, x_{n}}, \operatorname{Res}_{G_{x_{0}, \ldots, x_{n}}}^{G_{x_{0}}}\left(\mathfrak{l}_{x_{0}}\right)\right)
$$

for any $n \in \mathbb{N}$.

Proof. Let $\left[G / \operatorname{Sd}_{n}(X)\right]$ be a set of representative of the $G$-action on $\operatorname{Sd}_{n}(X)$. Let $x=$ $x_{0}<\ldots<x_{n}$ be a chain in $\operatorname{Sd}_{n}(X)$ then there exist some $g_{x} \in G$ and a unique $\sigma_{x} \in\left[G / \operatorname{Sd}_{n}(X)\right]$ such that $x=g_{x} \sigma_{x}$ where $\sigma_{x}=\sigma_{x_{0}}<\ldots<\sigma_{x_{n}}$. We define 


$$
(f, \lambda):\left(\operatorname{Sd}_{n}(X), \mathfrak{l}_{n}\right) \rightarrow \underset{x_{0}<\ldots<x_{n} \in G}{\operatorname{Sd}_{n}(X)}\left(G / G_{x_{0}, \ldots, x_{n}}, \operatorname{Res}_{G_{x_{0}, \ldots, x_{n}}}^{G_{x_{0}}}\left(\mathfrak{l}_{x_{0}}\right)\right)
$$

where $f(x)=g_{x} G_{\sigma_{x}} \in G / G_{\sigma_{x}}$ and $\lambda_{x}=\mathfrak{l}\left(g_{x}^{-1}, g_{x} \sigma_{x_{0}}, \sigma_{x_{0}}\right)$. Obviously,

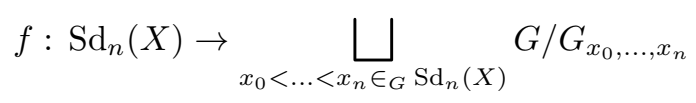

is an isomorphism of $G$-sets. We show that

$$
\lambda: \mathfrak{l}_{n} \rightarrow \bigsqcup_{x_{0}<\ldots<x_{n} \in G S d_{n}(X)} \operatorname{Res}_{G_{x_{0}, \ldots, x_{n}}}^{G_{x_{0}}}\left(\mathfrak{l}_{x_{0}}\right) \circ f
$$

is a natural transformation. Let $x=x_{0}<\ldots<x_{n}$, and $y=y_{0}<\ldots<y_{n}$ be sequences in $\operatorname{Sd}_{n}(X)$ such that $g x=y$ for some $g \in G$. There exist a unique $\sigma_{x}, \sigma_{y} \in\left[G / \operatorname{Sd}_{n}(X)\right]$ such that $x=g_{x} \sigma_{x}$ and $y=g_{y} \sigma_{y}$ for some $g_{x}$ and $g_{y}$ in $G$. Then $x_{0}=g_{x} \sigma_{x_{0}}$ and $y_{0}=g_{y} \sigma_{y_{0}}$ so $y_{0}=g x_{0}=g g_{x} \sigma_{x_{0}}$. Thus, by uniqueness $\sigma_{x_{0}}=\sigma_{y_{0}}$ and so $g_{y}^{-1} g g_{x} \in G_{\sigma_{x_{0}}}$. Then setting $r=\operatorname{Res}_{G_{x}}^{G_{x_{0}}}\left(\mathfrak{l}_{x_{0}}\right)(g, f(x), f(y)) \lambda_{x}$, we have that

$$
\begin{aligned}
r & =\widehat{\mathfrak{l}_{x_{0}}}\left(g, g_{x} G_{\sigma_{x}}, g_{y} G_{\sigma_{y}}\right) \mathfrak{l}\left(g_{x}^{-1}, g_{x} \sigma_{x_{0}}, \sigma_{x_{0}}\right)=\mathfrak{l}_{x_{0}}\left(g_{y}^{-1} g g_{x}\right) \mathfrak{l}\left(g_{x}^{-1}, g_{x} \sigma_{x_{0}}, \sigma_{x_{0}}\right) \\
& =\mathfrak{l}\left(g_{y}^{-1} g g_{x}, x_{0}, x_{0}\right) \mathfrak{l}\left(g_{x}^{-1}, g_{x} \sigma_{x_{0}}, \sigma_{x_{0}}\right) \\
& =\mathfrak{l}\left(g_{x}, \sigma_{x_{0}}, g_{x} \sigma_{x_{0}}\right) \mathfrak{l}\left(g, g_{x} \sigma_{x_{0}}, g g_{x} \sigma_{x_{0}}\right) \mathfrak{l}\left(g_{y}^{-1}, g g_{x} \sigma_{x_{0}}, \sigma_{x_{0}}\right) \mathfrak{l}\left(g_{x}^{-1}, g_{x} \sigma_{x_{0}}, \sigma_{x_{0}}\right) \\
& =\mathfrak{l}\left(g, x_{0}, y_{0}\right) \mathfrak{l}\left(g_{y}^{-1}, g_{y} \sigma_{y_{0}}, \sigma_{y_{0}}\right) \\
& =\mathfrak{l}_{n}(g, x, y) \lambda_{y} .
\end{aligned}
$$

By Remark 9, the Lefschetz invariant of a $C$-monomial $G$-set $(X, \mathfrak{l})$ can be written as

$$
\Lambda_{(X, \mathfrak{l})}=\sum_{x_{0}<\ldots<x_{n} \in G X}(-1)^{n}\left[G_{x_{0}, \ldots x_{n}}, \operatorname{Res}_{G_{x_{0}, \ldots, x_{n}}}^{G_{x_{0}}}\left(\mathfrak{l}_{x_{0}}\right)\right]_{G}=\sum_{n \in \mathbb{N}}(-1)^{n}\left(\operatorname{Sd}_{n}(X), \mathfrak{l}_{n}\right) .
$$

It follows that $\Lambda_{X}=\Lambda_{\tau_{G}(X)}$, where $\Lambda_{X}$ the Lefschetz invariant of the $G$-poset $X$ introduced in [2].

We define similarly the reduced Lefschetz invariant of $(X, \mathfrak{l})$

$$
\widetilde{\Lambda}_{(X, \mathfrak{l})}=\Lambda_{(X, \mathfrak{l})}-\left[G, 1_{G}\right]_{G}
$$

where $1_{G}$ is the trivial character of $G$.

Lemma 10. Let $G$ be a finite group and $C$ be an abelian group.

1. Let $(X, \mathfrak{l})$ be a $C$-monomial $G$-set, viewed as a $C$-monomial $G$-poset ordered by the equality relation on $X$. Then $\Lambda_{(X, \mathfrak{l})}=\left[C \times_{\mathfrak{l}} X\right]$ in $B_{C}(G)$. 
2. Let $(X, \mathfrak{l})$ and $(Y, \mathfrak{m})$ be $C$-monomial $G$-posets. Then $\Lambda_{(X \sqcup Y, \mathfrak{r})}=\Lambda_{(X, \mathfrak{l})}+\Lambda_{(Y, \mathfrak{m})}$ in $B_{C}(G)$.

3. Given $C$-monomial $G$-posets $(X, \mathfrak{l})$ and $(Y, \mathfrak{m})$, we have $\Lambda_{(X \times Y, \mathfrak{l} \times \mathfrak{m})}=\Lambda_{(X, \mathfrak{l})} \Lambda_{(Y, \mathfrak{m})}$ in $B_{C}(G)$.

Proof. 1. and 2. are clear.

3. In the following proof using the inclusion

$$
B_{C}(G) \hookrightarrow \mathbb{Q} \otimes_{\mathbb{Z}} B_{C}(G)
$$

we identify the elements of $B_{C}(G)$ with their image in $\mathbb{Q} \otimes_{\mathbb{Z}} B_{C}(G)$. We start with rearranging the chains in $X \times Y$ as in the proof of Lemma 11.2.9 in [2]. Let $n \in \mathbb{N}$. Given a chain $z=z_{0}<\ldots<z_{n}$ in $X \times Y$ projection of $z$ on $X$ is denoted by $z_{X}$ and on $Y$ is denoted by $z_{Y}$. Then $z_{X}$ is a chain in $X$ with order $i+1$ for some $i \leq n$ and $z_{Y}$ is a chain in $Y$ with order $j+1$ for some $j \leq n$ such that $i+j=n$. Let $\underline{s}_{i}$ be the chain $s_{0}<\ldots<s_{i}$ and $\underline{t}_{j}$ be the chain $t_{0}<\ldots<t_{j}$. Now

$$
\begin{aligned}
\Lambda_{(X \times Y, \mathfrak{l} \times \mathfrak{m})}= & \sum_{\substack{n \in \mathbb{N} \\
z \in G}}(-1)^{n}\left[G_{z}, \operatorname{Res}_{G_{z}}^{G_{z_{0}}}\left(\mathfrak{l}_{z_{0}}\right)\right]_{G} \\
= & \sum_{\substack{n \in \mathbb{N}, z \in \operatorname{Sd}_{n}(X \times Y)}}(-1)^{n} \frac{\left|G_{z}\right|}{|G|}\left[G_{z}, \operatorname{Res}_{G_{z}}^{G_{z_{0}}}\left(\mathfrak{l}_{z_{0}}\right)\right]_{G} \\
= & \sum_{\substack{i, j \in \mathbb{N} \\
\underline{s}_{i} \in X}} \Gamma_{\underline{s}_{i}, \underline{t}_{j}} \\
\underline{\underline{t}}_{j} \in Y &
\end{aligned}
$$

where

$$
\begin{aligned}
& \Gamma_{\underline{s}_{i}, \underline{t}_{j}}=\sum_{\substack{n \in \mathbb{N} \\
z \in \operatorname{Sd}_{n}(X \times Y): z_{X}=\underline{s}_{i}, z_{Y}=\underline{t}_{j}}}(-1)^{n} \frac{\left|G_{\underline{s}_{i}} \cap G_{\underline{t}_{j}}\right|}{|G|}\left[G_{\underline{s}_{i}} \cap G_{\underline{t}_{j}}, \operatorname{Res}_{G_{\underline{s}_{i}}}^{G_{s_{0}}}\left(\mathfrak{l}_{s_{0}}\right) \operatorname{Res}_{G_{\underline{t}_{j}}}^{G_{t_{0}}}\left(\mathfrak{m}_{t_{0}}\right)\right]_{G}
\end{aligned}
$$

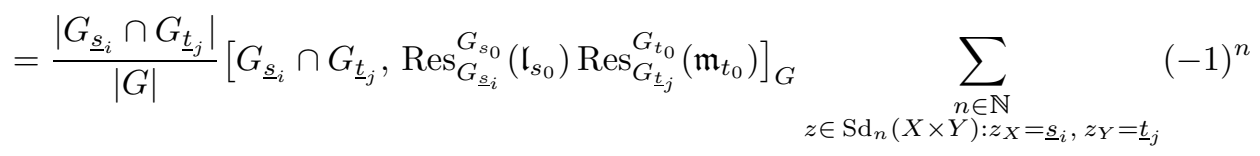

$$
\begin{aligned}
& =\frac{\left|G_{\underline{s}_{i}} \cap G_{\underline{t}_{j}}\right|}{|G|}\left[G_{\underline{s}_{i}} \cap G_{\underline{t}_{j}}, \operatorname{Res}_{G_{\underline{s}_{i}}}^{G_{s_{0}}}\left(\mathfrak{l}_{s_{0}}\right) \operatorname{Res}_{G_{\underline{t}_{j}}}^{G_{t_{0}}}\left(\mathfrak{m}_{t_{0}}\right)\right]_{G}(-1)^{i+j} .
\end{aligned}
$$

Now,

$$
\Lambda_{(X \times Y, \mathfrak{l} \times \mathfrak{m})}=\sum_{\substack{i, j \in \mathbb{N} \\ \underline{s}_{i} \in X \\ \underline{t}_{j} \in Y}}(-1)^{i+j} \frac{\left|G_{\underline{s}_{i}} \cap G_{\underline{t}_{j}}\right|}{|G|}\left[G_{\underline{s}_{i}} \cap G_{\underline{t}_{j}}, \operatorname{Res}_{G_{\underline{s}_{i}}}^{G_{s_{0}}}\left(\mathfrak{l}_{s_{0}}\right) \operatorname{Res}_{G_{\underline{t}_{j}}}^{G_{t_{0}}}\left(\mathfrak{m}_{t_{0}}\right)\right]_{G} .
$$


On the other hand

$$
\begin{aligned}
& \Lambda_{(X, \mathfrak{l})} \Lambda_{(Y, \mathfrak{m})}=\sum_{\substack{i \in \mathbb{N} \\
\underline{s}_{i} \in G}}(-1)\left[G_{\underline{s}_{i}}, \operatorname{Res}_{G_{\underline{s}_{i}}}^{G_{s_{0}}}\left(\mathfrak{l}_{s_{0}}\right)\right] \sum_{\substack{j \in \mathbb{N} \\
\underline{t}_{j} \in G}}(-1)^{j}\left[G_{\underline{t}_{j}}, \operatorname{Res}_{G_{\underline{t}_{j}}}^{G_{t_{0}}}\left(\mathfrak{m}_{t_{0}}\right)\right]_{G} \\
& =\sum_{\substack{i, j \in \mathbb{N} \\
\underline{s}_{i} \in X \\
\underline{\underline{t}}_{j} \in Y \\
G_{i} g G_{\underline{\underline{t}} j} \subseteq G}}(-1)^{i+j} \frac{\left|G_{\underline{s}_{i}}\right|\left|G_{\underline{t}_{j}}\right|}{|G|^{2}}\left[G_{\underline{s}_{i}} \cap{ }^{g} G_{\underline{t}_{j}}, \operatorname{Res}_{G_{\underline{s}_{i}}}^{G_{s_{0}}}\left(\mathfrak{l}_{s_{0}}\right) \operatorname{Res}_{g^{g}} G_{\underline{t}_{t_{j}}}\left({ }^{g} \mathfrak{m}_{t_{0}}\right)\right]_{G} \\
& =\sum_{\substack { i, j \in \mathbb{N} \\
\begin{subarray}{c}{\underline{s}_{i} \in X \\
\underline{t}_{j} \in Y \\
g \in G{ i , j \in \mathbb { N } \\
\begin{subarray} { c } { \underline { s } _ { i } \in X \\
\underline { t } _ { j } \in Y \\
g \in G } }\end{subarray}}(-1)^{i+j} \frac{\left|G_{\underline{s}_{i}}\right|\left|G_{\underline{t}_{j}}\right|}{|G|^{2}\left|G_{\underline{s}_{i}} g G_{\underline{t}_{j}}\right|}\left[G_{\underline{s}_{i}} \cap{ }^{g} G_{\underline{\underline{t}}_{j}}, \operatorname{Res}_{G_{\underline{s}_{i}}}^{G_{s_{0}}}\left(\mathfrak{l}_{s_{0}}\right) \operatorname{Res}_{g_{G_{\underline{t}_{j}}}}^{g} G_{t_{0}}\left({ }_{\mathfrak{m}_{t_{0}}}\right)\right]_{G} \\
& =\sum_{\substack{i, j \in \mathbb{N} \\
\underline{s}_{i} \in X \\
\underline{t}_{j} \in Y \\
g \in G}}(-1)^{i+j} \frac{\left|G_{\underline{s}_{i}} \cap{ }^{g} G_{\underline{t}_{j}}\right|}{|G|^{2}}\left[G_{\underline{s}_{i}} \cap{ }^{g} G_{\underline{t}_{j}}, \operatorname{Res}_{G_{\underline{s}_{i}}}^{G_{s_{0}}}\left(\mathfrak{l}_{s_{0}}\right) \operatorname{Res}_{g_{G_{\underline{t}_{j}}} G_{t_{0}}}\left({ }^{g} \mathfrak{m}_{t_{0}}\right)\right]_{G} \\
& =\sum_{\substack{i, j \in \mathbb{N} \\
\underline{s}_{i} \in X \\
\underline{t}_{j} \in Y \\
g \in G}}(-1)^{i+j} \frac{\left|G_{\underline{s}_{i}} \cap G_{g_{\underline{t}_{j}}}\right|}{|G|^{2}}\left[G_{\underline{s}_{i}} \cap G_{g_{\underline{t}_{j}}}, \operatorname{Res}_{G_{\underline{s}_{i}}}^{G_{s_{0}}}\left(\mathfrak{l}_{s_{0}}\right) \operatorname{Res}_{G_{g_{\underline{t}_{j}}}}^{G_{g_{t_{0}}}}\left(\mathfrak{m}_{g t_{0}}\right)\right]_{G} \\
& =\sum_{\substack{i, j \in \mathbb{N} \\
\underline{s}_{i} \in X}}(-1)^{i+j} \frac{\left|G_{\underline{s}_{i}} \cap G_{\underline{t}_{j}}\right|}{|G|}\left[G_{\underline{s}_{i}} \cap G_{\underline{t}_{j}}, \operatorname{Res}_{G_{\underline{s}_{i}}}^{G_{s_{0}}}\left(\mathfrak{l}_{s_{0}}\right) \operatorname{Res}_{G_{\underline{t}_{j}}}^{G_{t_{0}}}\left(\mathfrak{m}_{t_{0}}\right)\right]_{G} .
\end{aligned}
$$

Thus, $\Lambda_{(X \times Y, \mathfrak{l} \times \mathfrak{m})}=\Lambda_{(X, \mathfrak{l})} \Lambda_{(Y, \mathfrak{m})}$.

The first assertion of Lemma 10 tells us that every positive element of $B_{C}(G)$ is in of the form $\Lambda_{(X, \mathfrak{l})}$ for some $C$-monomial $G$-poset $(X, \mathfrak{l})$. Now consider the poset $X=$ $\{a, b, c, d, e\}$ with the ordering $\{a \leq c, a \leq d, a \leq e, b \leq c, b \leq d, b \leq e\}$. Consider trivial $G$-action on $X$. Then $\Lambda_{\tau_{G}(X)}=-1_{B_{C}(G)}$. So as a consequence of Lemma 10 we get the following corollary.

Corollary 11. Any element of the monomial Burnside ring can be expressed as the Lefschetz invariant of some (non unique) monomial G-poset.

Proposition 12. Let $H$ be a subgroup of $G$. Given a $C$-monomial $H$-poset $(X, \mathfrak{l})$, we have

$$
\operatorname{Ind}_{H}^{G}\left(\Lambda_{(X, \mathfrak{l})}\right)=\Lambda_{\operatorname{Ind}_{H}^{G}(X, \mathfrak{l})}
$$


Proof. Since

$$
\operatorname{Ind}_{H}^{G}\left(\Lambda_{(X, \mathfrak{l})}\right)=\sum_{n \in \mathbb{N}}(-1)^{n} \operatorname{Ind}_{H}^{G}\left(\operatorname{Sd}_{n}(X), \mathfrak{l}_{n}\right)
$$

we need to show that there exists a $C$-monomial $G$-set isomorphism between

$$
\left(G \times_{H} \operatorname{Sd}_{n}(X), G \times_{H} \mathfrak{l}_{n}\right)
$$

and

$$
\left(\operatorname{Sd}_{n}\left(G \times_{H} X\right),\left(G \times_{H} \mathfrak{l}\right)_{n}\right)
$$

for any $n \in \mathbb{N}$.

We define

$$
\left(f_{n}, \mathrm{id}\right):\left(G \times_{H} \operatorname{Sd}_{n}(X), G \times_{H} \mathfrak{l}_{n}\right) \rightarrow\left(\operatorname{Sd}_{n}\left(G \times_{H} X\right),\left(G \times_{H} \mathfrak{l}\right)_{n}\right)
$$

where

$$
f_{n}: G \times_{H} \operatorname{Sd}_{n}(X) \rightarrow \operatorname{Sd}_{n}\left(G \times_{H} X\right)
$$

such that

$$
f_{n}\left(u,{ }_{H} x_{0}<\ldots<x_{n}\right)=\left(\left(u,{ }_{H} x_{0}\right)<\ldots<\left(u,{ }_{H} x_{n}\right)\right)
$$

for any chain $\left(u,{ }_{H} x_{0}<\ldots<x_{n}\right)$ in $G \times_{H} \operatorname{Sd}_{n}(X)$.

Let $\left(u_{0},{ }_{H} x_{0}\right)<\ldots<\left(u_{n},{ }_{H} x_{n}\right)$ be a chain in $\operatorname{Sd}_{n}\left(G \times_{H} X\right)$. There exist some $h_{i} \in H$ such that $u_{i} h_{i}=u_{i+1}$ and $h_{i}^{-1} x_{i}<x_{i+1}$ for all $0 \leq i \leq n-1$. Then

$$
f_{n}\left(u_{0},{ }_{H} x_{0}<h_{0} x_{1}<\ldots<h_{0} \ldots h_{n-1} x_{n}\right)=\left(u_{0},{ }_{H} x_{0}\right)<\ldots<\left(u_{n},{ }_{H} x_{n}\right) .
$$

Obviously, $f_{n}$ is a map of $G$-sets and injective.

Now, we show that $G \times_{H} \mathfrak{l}_{n}=\left(G \times_{H} \mathfrak{l}\right)_{n} \circ f_{n}$. We consider an element $k \in G$, and chains $\left(u,{ }_{H} x_{0}<\ldots<x_{n}\right)$ in $G \times{ }_{H} \operatorname{Sd}_{n}(X)$ such that

$$
k\left(u,{ }_{H} x_{0}<\ldots<x_{n}\right)=\left(v,_{H} y_{0}<\ldots<y_{n}\right) .
$$

There exists some $h \in H$ such that $k u=v h$ and $h x_{i}=y_{i}$ for all $0 \leq i \leq n$. Then

$$
\begin{aligned}
(G & \left.\times_{H} \mathfrak{l}\right)_{n}\left(k, f_{n}\left(u,{ }_{H} x_{0}<\ldots<x_{n}\right), f_{n}\left(v,{ }_{H} y_{0}<\ldots<y_{n}\right)\right) \\
& =\left(G \times_{H} \mathfrak{l}\right)_{n}\left(k,\left(u,{ }_{H} x_{0}\right)<\ldots<\left(u,{ }_{H} x_{n}\right),\left(v,_{H} y_{0}\right)<\ldots<\left(v,_{H} y_{n}\right)\right) \\
& =\mathfrak{l}_{n}\left(h, x_{0}<\ldots<x_{n}, y_{0}<\ldots<y_{n}\right) \\
& =\left(G \times_{H} \mathfrak{l}_{n}\right)\left(k,\left(u,{ }_{H} x_{0}<\ldots<x_{n}\right),\left(v,_{H} y_{0}<\ldots<y_{n}\right)\right) .
\end{aligned}
$$


Let $(X, \mathfrak{l})$ be a $G$-poset and let $x \in X$. Then the pairs (]$x, \cdot\left[{ }_{X}, \mathfrak{l}_{>x}\right)$ and (]$\cdot, x\left[X, \mathfrak{l}^{<x}\right)$ are $C$-monomial $G_{x}$-posets where

$$
] x, \cdot[X=\{y \in X \mid x<y\}, \quad] \cdot, x[X=\{y \in X \mid y<x\}
$$

which are $G_{x}$-posets and $\mathfrak{l}_{>x}: \widehat{] x, \cdot[X} \rightarrow \bullet_{C}$ and $\mathfrak{l}<x: \widehat{:, x[X} \rightarrow \bullet_{C}$ are the restrictions of the functor $\mathfrak{l}$.

Lemma 13. Let $(X, \mathfrak{l})$ be a monomial $G$-poset. We have

$$
\Lambda_{(X, \mathfrak{l})}=-\sum_{x \in[G / X]} \operatorname{Ind}_{G_{x}}^{G}\left(\left[G_{x}, \mathfrak{l}_{x}\right]_{G_{x}} \cdot \widetilde{\Lambda}_{] x,[X}\right) .
$$

\section{Proof.}

$$
\begin{aligned}
\Lambda_{(X, \mathfrak{l})} & =\sum_{x_{0}<\ldots<x_{n} \in G X}(-1)^{n}\left[G_{x_{0}, \ldots, x_{n}}, \operatorname{Res}_{G_{x_{0}, \ldots, x_{n}}}^{G_{x_{0}}}\left(\mathfrak{l}_{x_{0}}\right)\right]_{G} \\
& =\sum_{x_{0} \in_{G} X} \sum_{x_{1}<\ldots<x_{n} \in G X: x_{0}<x_{1}}(-1)^{n}\left[G_{x_{0}, \ldots, x_{n}}, \operatorname{Res}_{G_{x_{0}, \ldots, x_{n}}}^{G_{x_{0}}}\left(\mathfrak{l}_{x_{0}}\right)\right]_{G} \\
& =\sum_{x_{0} \in \in_{G} X} \operatorname{Ind}_{G_{x_{0}}}^{G} \sum_{\left.x_{1}<\ldots<x_{n} \in_{G_{x_{0}}}\right] x_{0}, \cdot[X}(-1)^{n}\left[G_{x_{0}, \ldots, x_{n}}, \operatorname{Res}_{G_{x_{0}, \ldots, x_{n}}}^{G_{x_{0}}}\left(\mathfrak{l}_{x_{0}}\right)\right]_{G_{x_{0}}} \\
& =\sum_{x_{0} \in \in_{G} X} \operatorname{Ind}_{G_{x_{0}}}^{G}\left[G_{x_{0}}, \mathfrak{l}_{x_{0}}\right]_{G_{x_{0}}} \sum_{\left.x_{1}<\ldots<x_{n} \in \in_{G_{0}}\right] x_{0}, \cdot[X}(-1)^{n}\left[G_{x_{0}, \ldots, x_{n}}, 1_{G_{x_{0}, \ldots, x_{n}}}\right]_{G_{x_{0}}} \\
& =-\sum_{x \in \in_{G} X} \operatorname{Ind}_{G_{x}}^{G}\left(\left[G_{x}, \mathfrak{l}_{x}\right]_{G_{x}} \cdot \widetilde{\Lambda}_{] x,[X}\right) .
\end{aligned}
$$

Remark 14. We can define the opposite of a $C$-monomial $G$-poset $(X, \mathfrak{l})$ as follows. We consider the pair $\left(X^{\text {op }}, \mathfrak{l}^{\text {op }}\right)$ where $X^{\text {op }}$ is the opposite $G$-poset with the order $\leq$ op defined by

$$
\forall x, y \in X, g \in G, g x \leq^{\text {op }} y \Leftrightarrow y \leq g x
$$

and $\mathfrak{l}^{\mathrm{op}}: \widehat{X^{\mathrm{op}}} \rightarrow \bullet_{C}$ is defined by

$$
\mathfrak{l}^{\mathrm{op}}(g, x, y)=\mathfrak{l}^{-1}\left(g^{-1}, y, x\right)
$$

for any $x, y \in X^{\text {op }}$ and $g \in G$ such that $g x \leq$ op $y$. Obviously, the pair ( $X^{\text {op }}, \mathfrak{l}^{\text {op }}$ ) is a $C$-monomial $G$-poset. Moreover the assignment $(X, \mathfrak{l}) \mapsto\left(X^{\text {op }}, \mathfrak{l}\right.$ op $)$ is a functor ${ }_{C} M G$-poset $\rightarrow{ }_{C} M G$-poset: if $(f, \lambda):(X, \mathfrak{l}) \rightarrow(Y, \mathfrak{m})$ is a map of $C$-monomial $G$-posets, then $f: X^{\text {op }} \rightarrow Y^{\text {op }}$ is a map of $G$-posets and for any $g x \leq{ }^{\text {op }} x^{\prime}$, we get the commutative diagram 


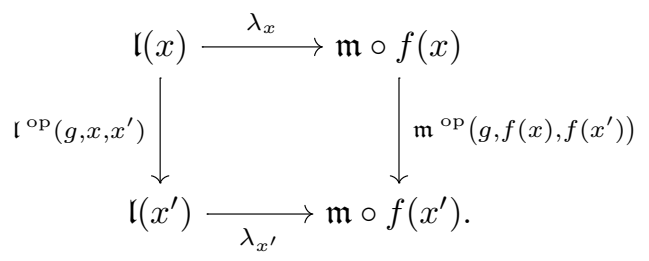

Observe that $(\mathfrak{l o p})_{x}(g)=\mathfrak{l}^{-1}\left(g^{-1}, x, x\right)=\mathfrak{l}(g, x, x)=\mathfrak{l}_{x}(g)$, for any $x \in X$ and $g \in G_{x}$. It follows that $\Lambda_{(X, \mathfrak{l})}=\Lambda_{(X \text { op }, \mathfrak{l o p})}$.

Let $(f, \lambda):(X, \mathfrak{l}) \rightarrow(Y, \mathfrak{m})$ be a map of $C$-monomial $G$-posets. Given $y \in Y$, following [3] we set

$$
f^{y}=\{x \in X \mid f(x) \leq y\}, \quad f_{y}=\{x \in X \mid f(x) \geq y\}
$$

which are both $G_{y}$-posets. We denote by $\left(f^{y}, \mathfrak{l}_{\mid f^{y}}\right)$ the $C$-monomial $G_{y}$-poset where $\mathfrak{l}_{\mid f^{y}}: \widehat{f^{y}} \rightarrow \bullet_{C}$ is the restriction of the functor $\mathfrak{l}$. Similarly, we denote by $\left(f_{y}, \mathfrak{l}_{\mid f_{y}}\right)$ to be $C$-monomial $G_{y}$-poset where $\mathfrak{l}_{\mid f_{y}}: \widehat{f}_{y} \rightarrow \bullet_{C}$ is the restriction of the functor $\mathfrak{l}$.

Example 15. Let $(f, \lambda):(X, \mathfrak{l}) \rightarrow(Y, \mathfrak{m})$ be a map of $C$-monomial $G$-posets. We define a $G$-poset $X *_{f, \lambda} Y$ with underlying $G$-set $X \sqcup Y$ as follows: for $z, z^{\prime} \in X \sqcup Y$, we set

$$
z \leq z^{\prime} \Leftrightarrow \begin{cases}z, z^{\prime} \in X & \text { and } \quad z \leq z^{\prime} \in X \\ z, z^{\prime} \in Y & \text { and } \quad z \leq z^{\prime} \in Y \\ z \in X, z^{\prime} \in Y & \text { and } \quad f(z) \leq z^{\prime} \in Y\end{cases}
$$

We define the functor $\mathfrak{l} *_{f, \lambda} \mathfrak{m}: \widehat{X \sqcup Y} \rightarrow \bullet_{C}$ by

$$
\left(\mathfrak{l} *_{f, \lambda} \mathfrak{m}\right)\left(g, z, z^{\prime}\right)= \begin{cases}\mathfrak{l}\left(g, z, z^{\prime}\right) & \text { if } z, z^{\prime} \in X \\ \mathfrak{m}\left(g, z, z^{\prime}\right) & \text { if } z, z^{\prime} \in Y \\ \mathfrak{m}\left(g, f(z), z^{\prime}\right) \lambda_{z} & \text { if } z \in X, z^{\prime} \in Y\end{cases}
$$

for any $z, z^{\prime} \in X *_{f, \lambda} Y$ and $g \in G$ such that $g z \leq z^{\prime}$.

Now let $z_{1}, z_{2}, z_{3} \in X *_{f, \lambda} Y$ and $g, g^{\prime} \in G$ such that $g z_{1} \leq z_{2}$ and $g^{\prime} z_{2} \leq z_{3}$. We aim to show that

$$
\left(\mathfrak{l} *_{f, \lambda} \mathfrak{m}\right)\left(g^{\prime} g, z_{1}, z_{3}\right)=\left(\mathfrak{l} *_{f, \lambda} \mathfrak{m}\right)\left(g^{\prime}, z_{2}, z_{3}\right)\left(\mathfrak{l} *_{f, \lambda} \mathfrak{m}\right)\left(g, z_{1}, z_{2}\right)
$$

We have four cases to consider:

- $z_{1}, z_{2}, z_{3} \in X$

- $z_{1}, z_{2} \in X$ and $z_{3} \in Y$, 
- $z_{1} \in X$ and $z_{2}, z_{3} \in Y$,

- $z_{1}, z_{2}, z_{3} \in Y$.

In the first case we get

$$
\begin{aligned}
\left(\mathfrak{l} *_{f, \lambda} \mathfrak{m}\right)\left(g^{\prime} g, z_{1}, z_{3}\right) & =\mathfrak{l}\left(g^{\prime} g, z_{1}, z_{3}\right)=\mathfrak{l}\left(g^{\prime}, z_{2}, z_{3}\right) \mathfrak{l}\left(g, z_{1}, z_{2}\right) \\
& =\left(\mathfrak{l} *_{f, \lambda} \mathfrak{m}\right)\left(g^{\prime}, z_{2}, z_{3}\right)\left(\mathfrak{l} *_{f, \lambda} \mathfrak{m}\right)\left(g, z_{1}, z_{2}\right)
\end{aligned}
$$

In the second case, using the naturality of $\lambda$ we get

$$
\begin{aligned}
& \left(\mathfrak{l} *_{f, \lambda} \mathfrak{m}\right)\left(g^{\prime} g, z_{1}, z_{3}\right)=\mathfrak{m}\left(g^{\prime} g, f\left(z_{1}\right), z_{3}\right) \lambda_{z_{1}}=\mathfrak{m}\left(g^{\prime}, f\left(z_{2}\right), z_{3}\right) \mathfrak{m}\left(g, f\left(z_{1}\right), f\left(z_{2}\right)\right) \lambda_{z_{1}} \\
& \quad=\mathfrak{l}\left(g, z_{1}, z_{2}\right) \mathfrak{m}\left(g^{\prime}, f\left(z_{2}\right), z_{3}\right) \lambda_{z_{2}}=\left(\mathfrak{l} *_{f, \lambda} \mathfrak{m}\right)\left(g^{\prime}, z_{2}, z_{3}\right)\left(\mathfrak{l} *_{f, \lambda} \mathfrak{m}\right)\left(g, z_{1}, z_{2}\right) .
\end{aligned}
$$

In the third case, we get

$$
\begin{aligned}
\left(\mathfrak{l} *_{f, \lambda} \mathfrak{m}\right)\left(g^{\prime} g, z_{1}, z_{3}\right) & =\mathfrak{m}\left(g^{\prime} g, f\left(z_{1}\right), z_{3}\right) \lambda_{z_{1}}=\mathfrak{m}\left(g^{\prime}, f\left(z_{2}\right), z_{3}\right) \mathfrak{m}\left(g, f\left(z_{1}\right), f\left(z_{2}\right)\right) \lambda_{z_{1}} \\
& =\left(\mathfrak{l} *_{f, \lambda} \mathfrak{m}\right)\left(g, z_{1}, z_{2}\right)\left(\mathfrak{l} *_{f, \lambda} \mathfrak{m}\right)\left(g^{\prime}, z_{2}, z_{3}\right)
\end{aligned}
$$

In the fourth case

$$
\begin{aligned}
\left(\mathfrak{l} *_{f, \lambda} \mathfrak{m}\right)\left(g^{\prime} g, z_{1}, z_{3}\right) & =\mathfrak{m}\left(g^{\prime} g, z_{1}, z_{3}\right)=\mathfrak{m}\left(g^{\prime}, z_{2}, z_{3}\right) \mathfrak{m}\left(g, z_{1}, z_{2}\right) \\
& =\left(\mathfrak{l} *_{f, \lambda} \mathfrak{m}\right)\left(g^{\prime}, z_{2}, z_{3}\right)\left(\mathfrak{l} *_{f, \lambda} \mathfrak{m}\right)\left(g, z_{1}, z_{2}\right) .
\end{aligned}
$$

Let $z \in X *_{f, \lambda} Y$ then obviously we have $\left(\mathfrak{l} *_{f, \lambda} \mathfrak{m}\right)(1, z, z)=1$. Thus, $\left(X *_{f, \lambda} Y, \mathfrak{l} *_{f, \lambda} \mathfrak{m}\right)$ is a $C$-monomial $G$-poset.

Lemma 16. Let $(f, \lambda):(X, \mathfrak{l}) \rightarrow(Y, \mathfrak{m})$ be a map of $C$-monomial $G$-posets. Then $\Lambda_{\left(X *_{f, \lambda} Y, \mathfrak{l}_{f, \lambda} \mathfrak{m}\right)}=\Lambda_{(Y, \mathfrak{m})}$.

Proof. 1. Let $z \in Z=X *_{f, \lambda} Y$. If $z \in X$ consider the map $\left.g:\right] z, \cdot[z \rightarrow[f(z), \cdot[Y$ defined by

$$
g(t)=\left\{\begin{array}{ll}
f(t) & \text { if } t \in X \\
t & \text { if } t \in Y
\end{array} .\right.
$$

Let $g^{\prime}:\left[f(z), \cdot[\rightarrow] z, \cdot\left[\right.\right.$ defined by $g^{\prime}(s)=s$. Then $g$ and $g^{\prime}$ are maps of $G_{z}$-posets such that $g \circ g^{\prime}=\mathrm{Id}$ and $\mathrm{Id} \leq g^{\prime} \circ g$. So if $z \in X$ using [[3], Lemma 4.2 .4 and Proposition 4.2.5], we get $\widetilde{\Lambda}_{] z, \cdot[}=\widetilde{\Lambda}_{[f(z), \cdot[}=0$. Thus, 


$$
\begin{aligned}
\Lambda_{\left(X *_{f, \lambda} Y, \mathfrak{l}_{f, \lambda} \mathfrak{m}\right)} & =-\sum_{z \in\left[G \backslash X *_{f, \lambda} Y\right]} \operatorname{Ind}_{G_{z}}^{G}\left(\left[G_{z}, \mathfrak{l}_{z}\right]_{G_{z}} \cdot \widetilde{\Lambda}_{] z, \cdot[}\right) \\
& =-\sum_{y \in[G \backslash Y]} \operatorname{Ind}_{G_{y}}^{G}\left(\left[G_{y}, \mathfrak{l}_{y}\right]_{G_{y}} \cdot \widetilde{\Lambda}_{] y, \cdot[}\right)=\Lambda_{(Y, \mathfrak{m})}
\end{aligned}
$$

As a consequence, we give an analogue of Proposition 4.2.7 in [3], which in turn was inspired by a much deeper theorem of Quillen in [6].

Proposition 17. Let $(f, \lambda):(X, \mathfrak{l}) \rightarrow(Y, \mathfrak{m})$ be a map of $C$-monomial $G$-posets. Then in $B_{C}(G)$

$$
\begin{aligned}
& \widetilde{\Lambda}_{(Y, \mathfrak{m})}=\widetilde{\Lambda}_{(X, \mathfrak{l})}+\sum_{y \in G \backslash Y} \operatorname{Ind}_{G_{y}}^{G}\left(\widetilde{\Lambda}_{f} \widetilde{\Lambda}_{(] y, \cdot[Y, \mathfrak{m}>y)}\right) . \\
& \widetilde{\Lambda}_{(Y, \mathfrak{m})}=\widetilde{\Lambda}_{(X, \mathfrak{l})}+\sum_{y \in G \backslash Y} \operatorname{Ind}_{G_{y}}^{G}\left(\widetilde{\Lambda}_{f_{y}} \widetilde{\Lambda}_{(], y\left[Y, \mathfrak{m}_{<y}\right)}\right) .
\end{aligned}
$$

Proof. We follow the proof of Proposition 4.2.7 in [3]. For any $n \in \mathbb{N}$, any chain $z=$ $z_{0}<\ldots<z_{n} \in \operatorname{Sd}_{n}\left(X *_{f, \lambda} Y\right)$ can be of two types, depending on $z_{n} \in X$ or $z_{n} \in Y$. For a sequence $z$ of the first type we get

$$
\operatorname{Res}_{G_{z_{0}, \ldots, z_{n}}}^{G_{z_{n}}}\left(\mathfrak{l} *_{f, \lambda} \mathfrak{m}\right)_{n_{z_{n}}}=\operatorname{Res}_{G_{z_{0}, \ldots, z_{n}}}^{G_{z_{n}}} \mathfrak{l}_{n}
$$

Now a sequence $z$ of the second type has a smallest element $y=z_{i}$ in $Y$, thus, we can write the sequence as

$$
x_{0}<\ldots<x_{i-1}<y<y_{0}<\ldots<y_{n-i-1}
$$

such that $x_{0}<\ldots<x_{i-1}$ is in $\operatorname{Sd}_{i-1}\left(f^{y}\right)$, and $y_{0}<\ldots<y_{n-i-1}$ is in $\operatorname{Sd}_{n-i-1}(] y, \cdot[Y)$. We get

$$
\operatorname{Res}_{G_{z_{0}, \ldots, z_{n}}}^{G_{z_{n}}}\left(\mathfrak{l} *_{f, \lambda} \mathfrak{m}\right)_{n_{z_{n}}}=\operatorname{Res}_{G_{z_{0}, \ldots, z_{n}}}^{G_{y}}(\mathfrak{m})
$$

Let $\underline{x}_{i-1}$ denote the chain $x_{0}<\ldots<x_{i-1}$ and $\underline{y}_{n-i-1}$ denote the chain $y_{0}<\ldots<y_{n-i-1}$. Then, by Lemma 10 and Lemma 16 we get

$$
\begin{aligned}
\Lambda_{(Y, \mathfrak{m})} & =\Lambda_{\left(X *_{f, \lambda} Y, \mathfrak{l}_{f, \lambda} \mathfrak{m}\right)}=\sum_{n \in \mathbb{N}}(-1)^{n}\left(\operatorname{Sd}_{n}\left(X *_{f, \lambda} Y\right),\left(\mathfrak{l} *_{f, \lambda} \mathfrak{m}\right)_{n}\right) \\
= & \sum_{\substack{n \in \mathbb{N} \\
z_{0}<\ldots<z_{n} \in \operatorname{Sd}_{n}\left(\mathfrak{l}_{f, \lambda} \mathfrak{m}\right)}}(-1)^{n}\left[G_{z_{0}, \ldots, z_{n}}, \operatorname{Res}_{G_{z_{0}, \ldots, z_{n}}}^{G_{z_{n}}}\left(\mathfrak{l} *_{f, \lambda} \mathfrak{m}\right)_{n}\right]_{G} \\
= & \sum_{n \in \mathbb{N}}(-1)^{n}\left(\operatorname{Sd}_{n}(X), \mathfrak{l}_{n}\right)
\end{aligned}
$$




$$
\begin{aligned}
& +\sum_{y \in[G \backslash Y]} \operatorname{Ind}_{G_{y}}^{G} \sum_{i=0}^{n} \sum_{\substack{\underline{x}_{i-1} \in \operatorname{Sd}_{i-1}\left(f^{y}\right) \\
\underline{y}_{n-i-1} \in \operatorname{Sd}_{n-i-1}(] y, \cdot\left[G_{y}\right)}}\left[G_{\underline{x}_{i-1}, y, \underline{y}_{n-i-1}}, \operatorname{Res}_{G_{\underline{x}_{i-1}, y, \underline{y}_{n-i-1}}}^{G_{y}} \mathfrak{m}_{y}\right]_{G_{y}} \\
& =\Lambda_{(X, \mathfrak{l})}+\sum_{y \in[G \backslash Y]} \operatorname{Ind}_{G_{y}}^{G}\left(\widetilde{\Lambda}_{\left(f^{y}, 1_{f} y\right)} \widetilde{\Lambda}_{(] y, \cdot\left[Y, \mathfrak{m}_{>y}\right)}\right) .
\end{aligned}
$$

For the second assertion we consider the opposite map

$$
(f, \lambda):\left(X^{\mathrm{op}}, \mathfrak{l}^{\mathrm{op}}\right) \rightarrow\left(Y^{\mathrm{op}}, \mathfrak{m}^{\mathrm{op}}\right)
$$

Since we have $\Lambda_{(X, \mathfrak{l})}=\Lambda_{(X \text { op }, \mathfrak{l o p})}$ by Remark 14, the result follows.

Corollary 18. Let $(f, \lambda):(X, \mathfrak{l}) \rightarrow(Y, \mathfrak{m})$ be a map of $C$-monomial $G$-posets. If $\Lambda_{f^{y}}=0$ for all $y \in Y$ (resp. if $\Lambda_{f_{y}}=0$ for all $y \in Y$ ), then $\Lambda_{X, \mathfrak{l}}=\Lambda_{Y, \mathfrak{m}}$.

Remark 19. The assumption of this corollary is fulfilled in particular if $f: \widehat{X} \rightarrow \widehat{Y}$ admits a right adjoint $g$, in other words if there exists a map of posets $g: Y \rightarrow X$ such that $f(x) \leq y \Leftrightarrow x \leq g(y)$ for any $x \in X$ and $y \in Y$, i.e. equivalently if $f \circ g(y) \leq y$ and $g \circ f(x) \leq x$ for any $x \in X$ and any $y \in Y$.

Now we set some notation. Given a $C$-monomial $G$-set $(X, \mathfrak{l})$, we can rewrite its Lefschetz invariant as

$$
\begin{aligned}
\Lambda_{(X, \mathfrak{l})} & =\sum_{x_{0}<\ldots<x_{n} \in G}(-1)^{n}\left[G_{x_{0}, \ldots x_{n}}, \operatorname{Res}_{G_{x_{0}, \ldots, x_{n}}}^{G_{x_{0}}}\left(\mathfrak{l}_{x_{0}}\right)\right]_{G} \\
& =\sum_{(V, \nu) \in_{G} \operatorname{ch}(G)} \gamma_{V, \nu}^{X, \mathfrak{l}}[V, \nu]_{G}
\end{aligned}
$$

where

$$
\begin{aligned}
\gamma_{V, \nu}^{X, \mathfrak{l}}= & \sum_{\substack{x_{0}<\ldots<x_{n} \in \in_{G} X \\
\left(G_{x_{0}, \ldots, x_{n}}, \operatorname{Res}_{G_{x_{0}}, \ldots, x_{n}} \mathfrak{l}_{x_{0}}\right)={ }_{G}(V, \nu)}}(-1)^{n} \\
= & \frac{1}{\left|N_{G}(V, \nu): V\right|} \sum_{\substack{\left.x_{0}<\ldots<x_{n} \in X \\
G_{x_{0}, \ldots, x_{n}}, \operatorname{Res}_{G_{x_{0}}, \ldots, x_{n}} \mathfrak{l}_{x_{0}}\right)=(V, \nu)}}(-1)^{n} .
\end{aligned}
$$

Given a $C$-monomial $G$-poset $(X, \mathfrak{l})$ we let the set $(X, \mathfrak{l})^{U, \mu}$ to be

$$
(X, \mathfrak{l})^{U, \mu}=\left\{x \in X^{U} \mid \operatorname{Res}_{U}^{G_{x}} \mathfrak{l}_{x}=\mu\right\}
$$

where $(U, \mu)$ is a subcharacter of $G$. Then given a $C$-subcharacter $(U, \mu) \in \operatorname{ch}(G)$ we have 


$$
\chi\left((X, \mathfrak{l})^{U, \mu}\right)=\sum_{\substack{n \in \mathbb{N} \\ x_{0}<\ldots<x_{n} \in X^{U} \\ \operatorname{Res}_{G_{0}} \mathfrak{x}_{x_{0}, \ldots, x_{n}} \mathfrak{l}_{x_{0}}=\mu}}(-1)^{n}=\sum_{\substack{(V, \nu) \in \operatorname{ch}(G) \\ U \subseteq V \\ \operatorname{Res}_{U}^{V} \nu=\mu}} m_{V, \nu}^{X, \mathfrak{l}}
$$

where

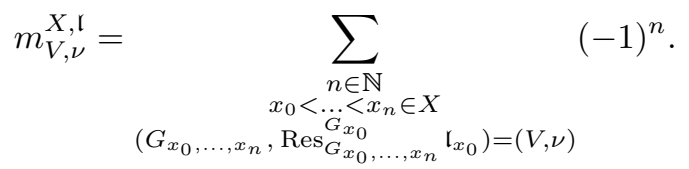

Now $\left|N_{G}(V, \nu): V\right| m_{V, \nu}^{X, \mathfrak{l}}=\gamma_{V, \nu}^{X, \mathfrak{l}}$. Using this fact we prove the following lemma.

Lemma 20. Let $(X, \mathfrak{l})$ and $(Y, \mathfrak{m})$ be C-monomial $G$-posets then $\Lambda_{(X, \mathfrak{l})}=\Lambda_{(Y, \mathfrak{m})}$ if and only if $\chi\left((X, \mathfrak{l})^{U, \mu}\right)=\chi\left((Y, \mathfrak{m})^{U, \mu}\right)$ for every $C$-subcharacter $(U, \mu)$ of $G$.

Proof. Assume $\Lambda_{(X, \mathfrak{l})}=\Lambda_{(Y, \mathfrak{m})}$. Then

$$
\begin{gathered}
\sum_{(V, \nu) \in_{G} \operatorname{ch}(G)} \gamma_{V, \nu}^{X, \mathfrak{l}}[V, \nu]_{G}=\sum_{(V, \nu) \in_{G} \operatorname{ch}(G)} \gamma_{V, \nu}^{Y, \mathfrak{m}}[V, \nu]_{G} \\
\sum_{(V, \nu) \in_{G} \operatorname{ch}(G)}\left(\gamma_{V, \nu}^{X, \mathfrak{l}}-\gamma_{V, \nu}^{Y, \mathfrak{m}}\right)[V, \nu]_{G}=0
\end{gathered}
$$

So $\gamma_{V, \nu}^{X, \mathfrak{l}}=\gamma_{V, \nu}^{Y, \mathfrak{m}}$ and then $m_{V, n u}^{X, \mathfrak{l}}=m_{V, n u}^{Y, \mathfrak{m}}$ for every $C$-subcharacter $(V, \nu)$ of $G$. We get

$$
\sum_{(U, \mu) \leq(V, \nu) \in_{G}} m_{V, \nu}^{X, \mathfrak{l}}=\sum_{(U, \mu) \leq(V, \nu) \in_{G} \operatorname{ch}(G)} m_{V, \nu}^{Y, \mathfrak{m}} .
$$

Thus, $\chi\left((X, \mathfrak{l})^{U, \mu}\right)=\chi\left((Y, \mathfrak{m})^{U, \mu}\right)$ for every $C$-subcharacter $(U, \mu)$ of $G$.

Conversely, assume that $\chi\left((X, \mathfrak{l})^{U, \mu}\right)=\chi\left((Y, \mathfrak{m})^{U, \mu}\right)$ for every $C$-subcharacter $(U, \mu)$ of $G$. Then

$$
\begin{gathered}
\sum_{(U, \mu) \leq(V, \nu) \in \operatorname{ch}(G)} m_{V, \nu}^{X, \mathfrak{l}}=\sum_{(U, \mu) \leq(V, \nu) \in \operatorname{ch}(G)} m_{V, \nu}^{Y, \mathfrak{m}}, \\
\sum_{(U, \mu) \leq(V, \nu) \in \operatorname{ch}(G)}\left(m_{V, \nu}^{X, \mathfrak{l}}-m_{V, \nu}^{Y, \mathfrak{m}}\right)=0 .
\end{gathered}
$$

Let $z$ be the matrix with the coefficients

$$
z(U, \mu ; V, \nu)=\lfloor(U, \mu) \leq(V, \nu)\rfloor= \begin{cases}1 & \text { if }(U, \mu) \leq(V, \nu) \\ 0 & \text { otherwise }\end{cases}
$$

for any $C$-subcharacters $(U, \mu),(V, \nu)$. If we list the $C$-subcharacters in non-decreasing order of size of the subgroups, the matrix $z$ is upper triangular with nonzero diagonal 
coefficients. Thus, $z$ is nonsingular and so $m_{V, \nu}^{X, \mathfrak{l}}=m_{V, \nu}^{Y, \mathfrak{m}}$. This implies $\gamma_{V, \nu}^{X, \mathfrak{l}}=\gamma_{V, \nu}^{Y, \mathfrak{m}}$. We get

$$
\Lambda_{(X, \mathfrak{l})}=\sum_{(V, \nu) \in_{G} \operatorname{ch}(G)} \gamma_{V, \nu}^{X, \mathfrak{l}}[V, \nu]_{G}=\sum_{(V, \nu) \in_{G} \operatorname{ch}(G)} \gamma_{V, \nu}^{Y, \mathfrak{m}}[V, \nu]_{G}=\Lambda_{(Y, \mathfrak{m})} .
$$

This proves the lemma.

\section{Generalized tensor induction}

Let $G$ and $H$ be finite groups. A set $U$ is a $(G, H)$-biset if $U$ is a left $G$-set and right $H$-set such that the $G$-action and the $H$-action commute. Any $(G, H)$-biset $U$ is a left $(G \times H)$-set with the following action:

$$
\forall u \in U,(g, h) \in G \times H(g, h) \cdot u=g u h^{-1} .
$$

A $C$-monomial $(G \times H)$-set $(U, \lambda)$ will be called a $C$-monomial $(G, H)$-biset, and usually denoted by $U_{\lambda}$ for simplicity.

Now let $U_{\lambda}$ be a $C$-monomial $(G \times H)$-set and $u, u^{\prime} \in U$. Then the set of morphisms from $u$ to $u^{\prime}$ in $\widehat{U}$ is

$$
\operatorname{Hom}_{\widehat{U}}\left(u, u^{\prime}\right)=\left\{(g, h) \in G \times H \mid g u=u^{\prime} h\right\}
$$

If $(g, h) \in \operatorname{Hom}_{\widehat{U}}\left(u, u^{\prime}\right)$, we denote the image of $(g, h)$ under $\lambda$ by $\lambda\left(g, h, u, u^{\prime}\right)$.

Let $U_{\lambda}$ be a $C$-monomial $(G, H)$-biset and $V_{\rho}$ be a $C$-monomial $(H, K)$-biset. Consider the set

$$
U_{\lambda} \circ V_{\rho}=\left\{(u, v) \in U \times V \mid \forall h \in H_{u} \cap H_{v}, \lambda(1, h, u, u) \rho(h, 1, v, v)=1\right\} .
$$

The set $U_{\lambda} \circ V_{\rho}$ is an $H$-set with the action

$$
\forall(u, v) \in U_{\lambda} \circ V_{\rho}, \forall h \in H, h(u, v)=\left(u h^{-1}, h v\right)
$$

Indeed, the condition that we impose on $U_{\lambda} \circ V_{\rho}$ amounts to saying that given $(u, v) \in$ $U_{\lambda} \circ V_{\rho}$, the linear character $\xi_{u, v}: h \mapsto \lambda(1, h, u, u) \rho(h, 1, v, v)$ of $H_{u} \cap H_{v}$ is trivial. Moreover we have $\xi_{u x, x^{-1} v}(h)=\xi_{u, v}\left(x h x^{-1}\right)=1$ for $x \in H$ and $h \in H_{u x} \cap H_{x^{-1} v}$, i.e. $x h x^{-1} \in H_{u} \cap H_{v}$.

We let $U_{\lambda} \circ_{H} V_{\rho}$ denote the set of $H$-orbits on $U_{\lambda} \circ V_{\rho}$ and $\left(u,{ }_{H} v\right)$ denote the $H$-orbit containing $(u, v)$. The set $U_{\lambda} \circ_{H} V_{\rho}$ is $(G, K)$-biset with the action

$$
\left(u,{ }_{H} v\right) \in U_{\lambda} \circ_{H} V_{\rho},(g, k) \in G \times K, g\left(u,{ }_{H} v\right) k=\left(g u,{ }_{H} v k\right) .
$$

We obtain a $C$-monomial $(G, K)$-biset $\left(U_{\lambda} \circ_{H} V_{\rho}, \lambda \times \rho\right)$, where $\lambda \times \rho$ is defined as follows: if $\left(u,{ }_{H} v\right)$ and $\left(u^{\prime},{ }_{H} v^{\prime}\right) \in U_{\lambda} \circ_{H} V_{\rho}$ and $(g, k) \in G \times K$ are such that $g\left(u,{ }_{H} v\right)=\left(u^{\prime},{ }_{H} v^{\prime}\right) k$, 
then there exists $h \in H$ such that $g u=u^{\prime} h$ and $h v=v^{\prime} k$. This element $h$ need not be unique, but it is well defined up to multiplication on the right by an element of $H_{u} \cap H_{v}$. We set

$$
(\lambda \times \rho)\left(g, k,\left(u,{ }_{H} v\right),\left(u^{\prime},{ }_{H} v^{\prime}\right)=\lambda\left(g, h, u, u^{\prime}\right) \rho\left(h, k, v, v^{\prime}\right)\right.
$$

which does not depend on the choice of $h$, by the defining property of $U_{\lambda} \circ V_{\rho}$. Note that $U_{\lambda} \circ_{H} V_{\rho}=U \times_{H} V$ when $V$ is a left free $(H, K)$-biset, or when $\lambda$ and $\rho$ are both equal to the trivial functor.

Given a $C$-monomial $G$-poset $(X, \mathfrak{l})$, we let $t_{U, \lambda}(X, \mathfrak{l})$ be the set of $G$-equivariant maps $f: U \rightarrow X$ such that

$$
\mathfrak{l}(g, f(u), f(u))=\lambda(g, 1, u, u)
$$

for all $u \in U$ and $g \in G_{u}$. Then $t_{U, \lambda}(X, \mathfrak{l})$ is an $H$-poset with the action $(h f)(u)=f(u h)$, for any $h \in H$, for any $f \in t_{U, \lambda}(X, \mathfrak{l})$, for any $u \in U$. The order $\leq$ is given as follows:

$$
\forall f, f^{\prime} \in t_{U, \lambda}(X, \mathfrak{l}), f \leq f^{\prime} \Leftrightarrow \forall u \in U, f(u) \leq f^{\prime}(u) \text { in } X
$$

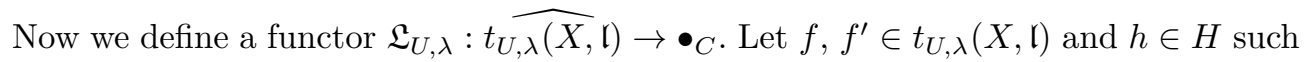
that $h f \leq f^{\prime}$. We choose a set $[G \backslash U]$ of representatives of $G$-orbits of $U$. Then for all $u \in U$ there exist some $g_{h, u} \in G$ and a unique $\sigma_{h}(u) \in[G \backslash U]$ such that

$$
u h=g_{h, u} \sigma_{h}(u) .
$$

Since $h f \leq f^{\prime}$, we get $g_{h, u} f\left(\sigma_{h}(u)\right) \leq f^{\prime}(u)$, and we set

$$
\mathfrak{L}_{U, \lambda}\left(h, f, f^{\prime}\right)=\prod_{u \in[G \backslash U]} \mathfrak{l}\left(g_{h, u}, f\left(\sigma_{h}(u)\right), f^{\prime}(u)\right) \lambda^{-1}\left(g_{h, u}, h, \sigma_{h}(u), u\right) .
$$

Now we show that this definition does not depend on the choice of $g_{h, u}$. Assume that there exist $g_{h, u}, g_{h, u}^{\prime} \in G$ such that

$$
u h=g_{h, u} \sigma_{h}(u)=g_{h, u}^{\prime} \sigma_{h}(u)
$$

So there exists $w \in G_{\sigma_{h}(u)}$ such that $g_{h, u}=g_{h, u}^{\prime} w$. We get

$$
\mathfrak{l}\left(w, f\left(\sigma_{h}(u)\right), f\left(\sigma_{h}(u)\right)\right)=\lambda\left(w, 1, \sigma_{h}(u), \sigma_{h}(u)\right) .
$$

Furthermore, we get the following commutative diagram:

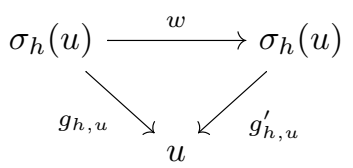


Thus,

$$
\begin{aligned}
\mathfrak{L}_{U, \lambda}\left(h, f, f^{\prime}\right) & =\prod_{u \in[G \backslash U]} \mathfrak{l}\left(g_{h, u}, f\left(\sigma_{h}(u)\right), f^{\prime}(u)\right) \lambda^{-1}\left(g_{h, u}, h, \sigma_{h}(u), u\right) \\
& =\prod_{u \in[G \backslash U]} \mathfrak{l}\left(g_{h, u}^{\prime} w, f\left(\sigma_{h}(u)\right), f^{\prime}(u)\right) \lambda^{-1}\left(g_{h, u}^{\prime} w, h, \sigma_{h}(u), u\right) \\
& =\prod_{u \in[G \backslash U]} \mathfrak{l}\left(g_{h, u}^{\prime}, f\left(\sigma_{h}(u)\right), f^{\prime}(u)\right) \lambda^{-1}\left(g_{h, u}^{\prime}, h, \sigma_{h}(u), u\right) .
\end{aligned}
$$

Definition 21. The above construction $T_{U, \lambda}:(X, \mathfrak{l}) \mapsto\left(t_{U, \lambda}(X, \mathfrak{l}), \mathfrak{L}_{U, \lambda}\right)$ is called the generalized tensor induction for $C$-monomial $G$-posets, associated to $(U, \lambda)$.

Lemma 22. Let $G$ and $K$ be finite groups and $U$ be $a(G, K)$-biset. Then there exists a bijection between the sets $\left\{(u, t) \mid u \in[G \backslash U / K], t \in\left[\left(K \cap G^{u}\right) \backslash K\right]\right\}$ and $[G \backslash U]$.

Proof. Let $u \in[G \backslash U / K]$ and $t \in\left[\left(K \cap G^{u}\right) \backslash K\right]$ then there exist some $g_{t, u} \in G$ and a unique $\sigma_{t}(u) \in[G \backslash U]$ such that

$$
u t=g_{t, u} \sigma_{t}(u)
$$

We define $\psi:\left\{(u, t) \mid u \in[G \backslash U / K], t \in\left[\left(K \cap G^{u}\right) \backslash K\right]\right\} \rightarrow[G \backslash U]$ by $\psi(u, t)=\sigma_{t}(u)$.

Lemma 23. Let $G$ and $H$ be finite groups, $(U, \lambda)$ be a monomial $(G, H)$-biset and $(X, \mathfrak{l})$ be a $C$-monomial $G$-poset.

1. $\left(t_{U, \lambda}(X, \mathfrak{l}), \mathfrak{L}_{U, \lambda}\right)$ is a $C$-monomial $H$-poset.

2. $\left(t_{U, \lambda}(X, \mathfrak{l}), \mathfrak{L}_{U, \lambda}\right)$ does not depend on the choice of representative set $[G \backslash U]$, up to isomorphism.

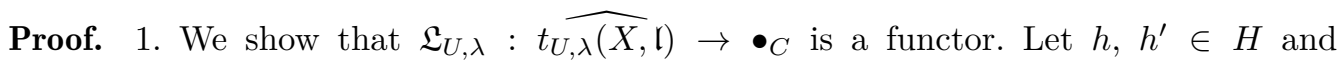
$f, f^{\prime}, f^{\prime \prime} \in t_{U, \lambda}(X, \mathfrak{l})$ such that $h f \leq f^{\prime}$ and $h^{\prime} f^{\prime} \leq f^{\prime \prime}$. Let $u \in[G \backslash U]$. Then there exist some $g_{h, u}, g_{h^{\prime}, u}, g_{h^{\prime} h, u}$ in $G$ and unique elements $\sigma_{h}(u), \sigma_{h^{\prime}}(u), \sigma_{h^{\prime} h}(u)$ in $[G \backslash U]$ such that

$$
u h=g_{h, u} \sigma_{h}(u), u h^{\prime}=g_{h^{\prime}, u} \sigma_{h^{\prime}}(u), u h^{\prime} h=g_{h^{\prime} h, u} \sigma_{h^{\prime} h}(u) .
$$

Also there exist some $g_{h, \sigma_{h^{\prime}}(u)} \in G$ and a unique $\sigma_{h}\left(\sigma_{h^{\prime}}(u)\right) \in[G \backslash U]$ such that

$$
\sigma_{h^{\prime}}(u) h=g_{h, \sigma_{h^{\prime}}(u)} \sigma_{h}\left(\sigma_{h^{\prime}}(u)\right)
$$

Now we get

$$
u h^{\prime} h=g_{h^{\prime}, u} g_{h, \sigma_{h^{\prime}}(u)} \sigma_{h}\left(\sigma_{h^{\prime}}(u)\right)
$$


and

$$
\sigma_{h^{\prime} h}(u)=\sigma_{h}\left(\sigma_{h^{\prime}}(u)\right)
$$

Then there exists $w \in G_{\sigma_{h^{\prime} h}(u)}$ such that

$$
g_{h^{\prime} h, u}=g_{h^{\prime}, u} g_{h, \sigma_{h^{\prime}}(u)} w .
$$

We have the following commutative diagram:

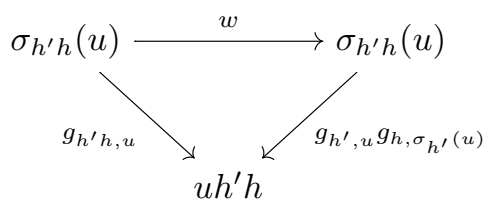

On the other hand since $w \in G_{\sigma_{h^{\prime} h}(u)}$, we get

$$
\mathfrak{l}\left(w, f\left(\sigma_{h^{\prime} h}(u)\right), f\left(\sigma_{h^{\prime} h}(u)\right)\right)=\lambda\left(w, 1, \sigma_{h^{\prime} h}(u), \sigma_{h^{\prime} h}(u)\right) .
$$

Thus, setting $L=\mathfrak{L}_{U, \lambda}\left(h^{\prime} h, f, f^{\prime \prime}\right)$, we have

$$
\begin{aligned}
L & =\prod_{u \in[G \backslash U]} \mathfrak{l}\left(g_{h^{\prime} h, u}, f\left(\sigma_{h^{\prime} h}(u)\right), f^{\prime \prime}(u)\right) \lambda^{-1}\left(g_{h^{\prime} h, u}, h^{\prime} h, \sigma_{h^{\prime} h}(u), u\right) \\
& =\prod_{u \in[G \backslash U]} \mathfrak{l}\left(g_{h^{\prime}, u} g_{h, \sigma_{h^{\prime}}}(u)\right. \\
& \left.=\prod_{u \in[G \backslash U]} \mathfrak{l}\left(g_{h^{\prime}, u} g_{h, \sigma_{h^{\prime}}(u)}, f\left(\sigma_{h^{\prime} h}(u)\right), f^{\prime \prime}(u)\right) \lambda^{-1}\left(g_{h^{\prime}, u}(u)\right), f_{h, \sigma_{h^{\prime}}(u)} w, h^{\prime} h, \sigma_{h^{\prime} h}(u), u\right) \\
& =\mathfrak{L}\left(h^{\prime}, f^{\prime}, f^{\prime \prime}\right) \mathfrak{L}\left(h, f, f^{\prime}\right) .
\end{aligned}
$$

Moreover, given $f \in T_{U, \lambda}(X, \mathfrak{l})$ we have

$$
\mathfrak{L}(1, f, f)=\prod_{u \in[G \backslash U]} \mathfrak{l}(1, f(u), f(u)) \lambda^{-1}(1,1, u, u)=1 .
$$

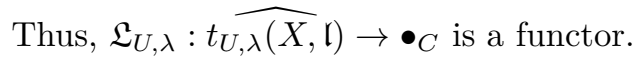

2. Let $h \in H$ and $f, f^{\prime} \in t_{U, \lambda}(X, \mathfrak{l})$ such that $h f \leq f^{\prime}$. Let $S=[G \backslash U]$ and let $S^{\prime}$ be the another choice of representatives. If $u^{\prime} \in S^{\prime}$ then there exist some $a_{u} \in G$, and a unique $u \in S$ such that $u^{\prime}=a_{u} u$. Then there exist some $g_{h, a_{u} u}, g_{h, u} \in G$, a unique $\sigma_{h}^{\prime}\left(a_{u} u\right) \in S^{\prime}$, and a unique $\sigma_{h}(u) \in S$ such that

$$
a_{u} u h=g_{h, a_{u} u} \sigma_{h}^{\prime}\left(a_{u} u\right)
$$


and

$$
u h=g_{h, u} \sigma_{h}(u)
$$

Then

$$
a_{u} u h=a_{u} g_{h, u} \sigma_{h}(u)=a_{u} g_{h, u} a_{\sigma_{h}(u)}^{-1} a_{\sigma_{h}(u)} \sigma_{h}(u) .
$$

So $\sigma_{h}^{\prime}\left(a_{u} u\right)=a_{\sigma_{h}(u)} \sigma_{h}(u)$. Note that $a_{\sigma_{h}(u)} \sigma_{h}(u) \in S^{\prime}$. We get the following commutative diagram:

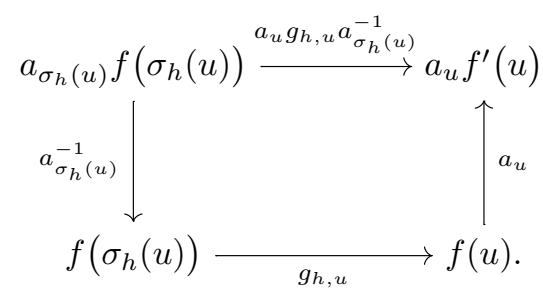

Thus, setting $L=\mathfrak{L}_{U, \lambda}^{\prime}\left(h, f, f^{\prime}\right)$, we have

$$
\begin{aligned}
L= & \prod_{a_{u} u \in S^{\prime}} \mathfrak{l}\left(a_{u} g_{h, u} a_{\sigma_{h}(u)}^{-1}, f\left(a_{\sigma_{h}(u)} \sigma_{h}(u)\right), f^{\prime}\left(a_{u} u\right)\right) \\
& \prod_{\lambda^{-1}\left(a_{u} g_{h, u} a_{\sigma_{h}(u)}^{-1}, h, a_{\sigma_{h}(u)} \sigma_{h}(u), a_{u} u\right)} \\
= & \mathfrak{L}_{U, \lambda}^{\prime}\left(h, f, f^{\prime}\right)=\mathfrak{L}_{U, \lambda}\left(h, f, f^{\prime}\right) \alpha_{f^{\prime}} \alpha_{f}^{-1}
\end{aligned}
$$

where

$$
\alpha_{f^{\prime}}=\prod_{u \in S} \mathfrak{l}\left(a_{u}, f^{\prime}(u), a_{u} f^{\prime}(u)\right) \lambda^{-1}\left(a_{u}, 1, u, a_{u} u\right)
$$

and

$$
\alpha_{f}^{-1}=\prod_{u \in S} \mathfrak{l}\left(a_{u}^{-1}, a_{u} f(u), f(u)\right) \lambda^{-1}\left(a_{u}^{-1}, 1, a_{u} u, u\right)
$$

Proposition 24. Let $G$ and $H$ be finite groups and $(U, \lambda)$ be a $C$-monomial $(G, H)$-biset.

1. Let $(X, \mathfrak{l}),\left(X^{\prime}, \mathfrak{l}^{\prime}\right)$ be $C$-monomial $G$-posets then

$$
T_{U, \lambda}\left((X, \mathfrak{l}) \times\left(X^{\prime}, \mathfrak{l}^{\prime}\right)\right) \cong T_{U, \lambda}(X, \mathfrak{l}) \times T_{U, \lambda}\left(X^{\prime}, \mathfrak{l}^{\prime}\right)
$$

2. $T_{U, \lambda}:{ }_{C} M G$-poset $\rightarrow{ }_{C} M H$-poset is a functor. 
Proof. 1. is clear.

2. Let $(\varphi, \beta):(X, \mathfrak{l}) \rightarrow(Y, \mathfrak{m})$ be a map of $C$-monomial $G$-posets. We define a map of $C$-monomial $G$-posets

$$
\left(T_{U, \lambda}(\varphi), T_{U, \lambda}(\beta)\right):\left(t_{U, \lambda}(X, \mathfrak{l}), \mathfrak{L}\right) \rightarrow\left(t_{U, \lambda}(Y, \mathfrak{m}), \mathfrak{M}\right)
$$

where

$$
T_{U, \lambda}(\varphi): t_{U, \lambda}(X, \mathfrak{l}) \rightarrow t_{U, \lambda}(Y, \mathfrak{m})
$$

such that $T_{U, \lambda}(\varphi)(f)=\varphi \circ f$ and

$$
T_{U, \lambda}(\beta): \mathfrak{L}(f) \rightarrow \mathfrak{M} \circ T_{U, \lambda}(\varphi)(f)
$$

such that

$$
T_{U, \lambda}(\beta)=\prod_{u \in[G \backslash U]} \beta_{f(u)}
$$

for any $f \in t_{U, \lambda}(X, \mathfrak{l})$. Clearly, $\varphi \circ f: U \rightarrow X \rightarrow Y$ is a map of $G$-posets. Since given $g \in G_{u}$ and $u \in U$ the map $\beta: \mathfrak{l} \rightarrow \mathfrak{m} \circ \varphi$ is natural, we have the following commutative diagram:

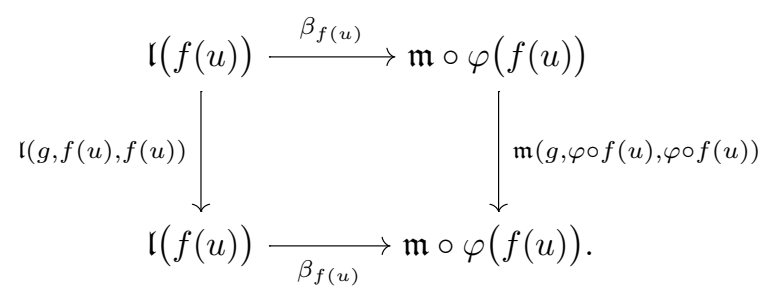

So

$$
\beta_{f(u)} \mathfrak{l}(g, f(u), f(u))=\mathfrak{m}(g, \varphi(f(u)), \varphi(f(u))) \beta_{f(u)}
$$

Since $g \in G_{f(u)}$, we have

$$
\mathfrak{l}(g, f(u), f(u))=\lambda(g, 1, u, u)
$$

Then we get

$$
\mathfrak{m}(g, \varphi(f(u)), \varphi(f(u)))=\lambda(g, 1, u, u)
$$

Thus, $\varphi \circ f \in t_{U, \lambda}(Y, \mathfrak{m})$. 
Now we show that

$$
T_{U, \lambda}(\beta): \mathfrak{L} \rightarrow \mathfrak{M} \circ T_{U, \lambda}(\varphi)
$$

is a natural transformation. Let $f, f^{\prime} \in t_{U, \lambda}(X, \mathfrak{l})$ and $h \in H$ such that $h f \leq f^{\prime}$. We show that the following diagram is commutative:

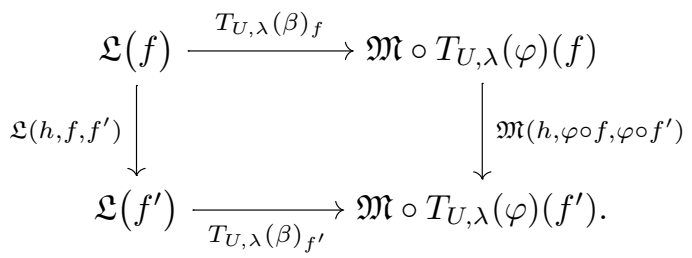

Let $u \in[G \backslash U]$. Then there exist some $g_{h, u} \in G$ and a unique $\sigma_{h}(u) \in[G \backslash U]$ such that

$$
u h=g_{h, u} \sigma_{h}(u) .
$$

Since $\beta: \mathfrak{l} \rightarrow \mathfrak{m} \circ \varphi$ is a natural transformation, we obtain the following commutative diagram:

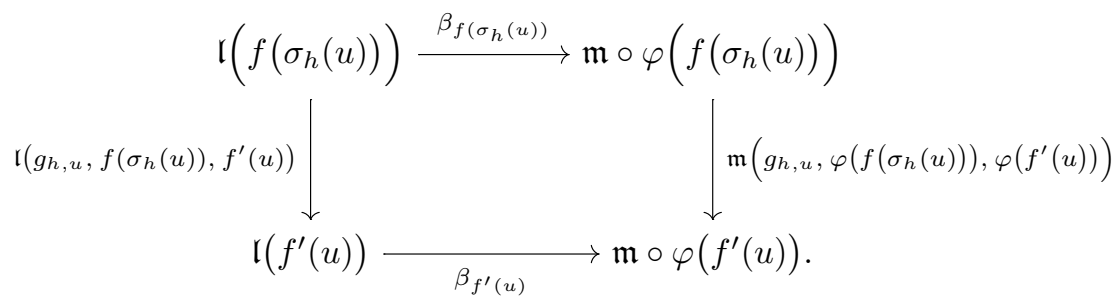

Using the commutativity of the above diagram, and setting $T=T_{U, \lambda}(\beta)_{f^{\prime}} \circ \mathfrak{L}\left(h, f, f^{\prime}\right)$, we get

$$
\begin{aligned}
T & =T_{U, \lambda}(\beta)_{f^{\prime}}\left(\prod_{u \in[G \backslash U]} \mathfrak{l}\left(g_{h, u}, f\left(\sigma_{h}(u)\right), f^{\prime}(u)\right) \lambda^{-1}\left(g_{h, u}, h, \sigma_{h}(u), u\right)\right) \\
& =\prod_{u \in[G \backslash U]} \beta_{f^{\prime}(u)} \mathfrak{l}\left(g_{h, u}, f\left(\sigma_{h}(u)\right), f^{\prime}(u)\right) \lambda^{-1}\left(g_{h, u}, h, \sigma_{h}(u), u\right) \\
& =\prod_{u \in[G \backslash U]} \mathfrak{m}\left(g_{h, u}, \varphi\left(f\left(\sigma_{h}(u)\right)\right), \varphi\left(f^{\prime}(u)\right)\right) \lambda^{-1}\left(g_{h, u}, h, \sigma_{h}(u), u\right) \beta_{f\left(\sigma_{h}(u)\right)} \\
& =\mathfrak{M}\left(h, \varphi \circ f, \varphi \circ f^{\prime}\right) \beta_{f} .
\end{aligned}
$$

So $T_{U, \lambda}(\beta): \mathfrak{L} \rightarrow \mathfrak{M} \circ T_{U, \lambda}(\varphi)$ is a natural transformation. Thus,

$$
\left(T_{U, \lambda}(\varphi), T_{U, \lambda}(\beta)\right):\left(t_{U, \lambda}(X, \mathfrak{l}), \mathfrak{L}\right) \rightarrow\left(t_{U, \lambda}(Y, \mathfrak{m}), \mathfrak{M}\right)
$$

is a map of $C$-monomial $G$-posets. 
Lemma 25. Let $G, H$ and $K$ be finite groups. If $U$ is a $(G, H)$-biset and $V$ is a left free $(H, K)$-biset, then the map $(u, v) \in U \times V \mapsto\left(u,{ }_{H} v\right) \in U \times{ }_{H} V$ restricts to a bijection $\pi:[G \backslash U] \times[H \backslash V] \rightarrow\left[G \backslash\left(U \times_{H} V\right)\right]$, where brackets denote sets of representatives of orbits.

Proof. For $(u, v) \in U \times V$, there exists $v_{0} \in[G \backslash V]$ and $h \in H$ such that $v=h v_{0}$. Then there exists $u_{0} \in[G \backslash U]$ and $g \in G$ such that $u h=g u_{0}$. Then $\left(u,{ }_{H} v\right)=g\left(u_{0},{ }_{H} v_{0}\right)$. Hence $\pi$ is surjective. Now if $\left(u_{0}, v_{0}\right)$ and $\left(u_{1}, v_{1}\right)$ are pairs in $[G \backslash U] \times[H \backslash V]$ which lie in the same $G$-orbit, there exists $g \in G$ and $h \in H$ such that $\left(g u_{0}, v_{0}\right)=\left(u_{1} h^{-1}, h v_{1}\right)$. Hence $h v_{1}=v_{0}$, so $v_{0}=v_{1}=h v_{1}$, and $h=1$ since $H$ act freely on $V$. Then $g u_{0}=u_{1}$, so $u_{0}=u_{1}$, and $\pi$ is injective.

Proposition 26. Let $G, H$ and $K$ be finite groups.

1. Let $(\bullet, 1)$ be the $C$-monomial $G$-poset where $\bullet$ is $G$-poset with one element and 1 : $\bullet \rightarrow \bullet_{C}$ is the functor such that $1(g, \bullet, \bullet)=1$. Then $T_{U, \lambda}(\bullet, 1)=(\bullet, 1)$.

2. Let $(\emptyset, z)$ be the empty $C$-monomial $(G, H)$-poset. Then $T_{\emptyset, z}$ is the constant functor with value $(\bullet, 1)$.

3. Let $(U, \lambda)$ and $\left(U^{\prime}, \lambda^{\prime}\right)$ be $C$-monomial $(G, H)$-bisets and let $(X, \mathfrak{l})$ be a $C$-monomial G-poset then

$$
T_{U \sqcup U^{\prime}, \lambda \sqcup \lambda^{\prime}}(X, \mathfrak{l})=T_{U, \lambda}(X, \mathfrak{l}) T_{U^{\prime}, \lambda^{\prime}}(X, \mathfrak{l}) .
$$

4. Let $\operatorname{id}_{G}$ stand for the identity $(G, G)$-biset. Then $T_{\operatorname{id}_{G}, 1}(X, \mathfrak{l})=(X, \mathfrak{l})$ for any $C$-monomial $G$-poset $(X, \mathfrak{l})$.

5. Let $(V, \rho)$ be a $C$-monomial left free $(H, K)$-biset, and $(U, \lambda)$ be a $C$-monomial $(H, G)$-biset. Then

$$
T_{V, \rho} \circ T_{U, \lambda}=T_{U \times_{H} V, \lambda \times \rho}
$$

Proof. 1., 2., 3. and 4. are clear.

5. Note that since $V$ is left free, we have $U_{\lambda} \circ_{H} V_{\rho} \cong{ }_{G} U \times{ }_{H} V_{K}$. Let $(X, \mathfrak{l})$ be a $C$-monomial $G$-poset. We need to show that

$$
\left(t_{V, \rho}\left(t_{U, \lambda}(X, \mathfrak{l}), \mathfrak{L}_{U, \lambda}\right), \mathfrak{L}_{V, \rho} \circ \mathfrak{L}_{U, \lambda}\right)=\left(t_{U \times_{H} V, \lambda \times \rho}(X, \mathfrak{l}), \mathfrak{L}_{U \times_{H} V, \lambda \times \rho}\right) .
$$

We define a $K$-poset map $\varphi: t_{V, \rho}\left(t_{U, \lambda}(X, \mathfrak{l}), \mathfrak{L}_{U, \lambda}\right) \rightarrow t_{U \times_{H} V, \lambda \times \rho}(X, \mathfrak{l})$ such that

$$
\varphi(f)\left(u,{ }_{H} v\right)=f(v)(u)
$$

for any $f \in t_{V, \rho}\left(t_{U, \lambda}(X, \mathfrak{l}), \mathfrak{L}_{U, \lambda}\right)$ and $\left(u,{ }_{H} v\right) \in U \times_{H} V$. It's clear that the map $\varphi(f)$ is a map of $G$-posets. 
Let $g \in G_{(u, H v)}$. Note that since $V$ is $H$-free, we have $g \in G_{u}$. Then

$$
\begin{aligned}
& \mathfrak{l}\left(g, \varphi(f)\left(u,{ }_{H} v\right), \varphi(f)\left(u,{ }_{H} v\right)\right)=\mathfrak{l}(g, f(v)(u), f(v)(u)) \\
& \quad=\lambda(g, 1, u, u) \rho(1,1, v, v)=(\lambda \times \rho)\left(g, 1,\left(u,{ }_{H} v\right),\left(u,{ }_{H} v\right)\right) .
\end{aligned}
$$

and so $\varphi(f) \in t_{U \times{ }_{H} V, \lambda \times \rho}(X, \mathfrak{l})$.

Now we define a map

$$
\theta: t_{U \times_{H} V, \lambda \times \rho}(X, \mathfrak{l}) \rightarrow t_{V, \rho}\left(t_{U, \lambda}(X, \mathfrak{l}), \mathfrak{L}_{U, \lambda}\right)
$$

such that $\theta(t)(v)(u)=t\left(u,{ }_{H} v\right)$ for any $t \in t_{U \times_{H} V, \lambda \times \rho}(X, \mathfrak{l}), u \in U$ and $v \in V$. We show that $\theta(t) \in t_{V, \rho}\left(t_{U, \lambda}(X, \mathfrak{l}), \mathfrak{L}_{U, \lambda}\right)$. Indeed, the map $\theta(t)$ is clearly a map of $H$-sets and moreover, since $V$ is $H$-free, we have $H_{v}=1$ for any $v \in V$. Then

$$
\mathfrak{L}_{U, \lambda}(1, \theta(t)(v), \theta(t)(v))=1=\rho(1,1, v, v) .
$$

Clearly, $\theta(t)(v)$ is a map of $G$-sets. Let $g \in G_{u}$. Then $g \in G_{(u, H} v$, and we get

$$
\begin{aligned}
\mathfrak{l}(g, \theta(t)(v)(u), \theta(t)(v)(u)) & =\mathfrak{l}\left(g, t\left(u,{ }_{H} v\right), t\left(u,{ }_{H} v\right)\right) \\
=\lambda(g, 1, u, u) \rho(1,1, v, v) & =\lambda(g, 1, u, u) .
\end{aligned}
$$

So $\theta(t) \in t_{V, \rho}\left(t_{U, \lambda}(X, \mathfrak{l}), \mathfrak{L}_{U, \lambda}\right)$.

Now we show that $\mathfrak{L}_{V, \rho} \circ \mathfrak{L}_{U, \lambda}=\mathfrak{L}_{U \times{ }_{H} V, \lambda \times \rho}$. Let $k \in K$ and $f, f^{\prime} \in t_{V, \rho}\left(t_{U, \lambda}(X, \mathfrak{l}), \mathfrak{L}_{U, \lambda}\right)$ such that $k f \leq f^{\prime}$. Let $v \in[H \backslash V]$. Then there exist a unique $\sigma_{k}(v) \in[H \backslash V]$ and some $h_{k, v} \in H$ such that

$$
v k=h_{k, v} \sigma_{k}(v) .
$$

Let $u \in[G \backslash U]$. Then there exist a unique $\sigma_{h_{k, v}}(u) \in[G \backslash U]$ and some $g_{h_{k, v}, u} \in G$ such that

$$
u h_{k, v}=g_{h_{k, v}, u} \sigma_{h_{k, v}}(u) .
$$

Then

$$
\begin{aligned}
\left(u,_{H} v\right) & =\left(u h_{k, v} h_{k, v}{ }^{-1},{ }_{H} v\right)=\left(u h_{k, v},_{H} h_{k, v}{ }^{-1} v\right) \\
& =\left(g_{h_{k, v}, u} \sigma_{h_{k, v}}(u){ }_{H} \sigma_{k}(v) k^{-1}\right)=g_{h_{k, v}, u}\left(\sigma_{h_{k, v}}(u),_{H} \sigma_{k}(v)\right) k^{-1} .
\end{aligned}
$$

We get

$$
\left(u,{ }_{H} v\right) k=g_{h_{k, v}, u}\left(\sigma_{h_{k, v}}(u),{ }_{H} \sigma_{k}(v)\right) .
$$


Then

$$
\left(\sigma_{h_{k, v}}(u),{ }_{H} \sigma_{k}(v)\right)=\sigma_{k}\left(u,{ }_{H} v\right)
$$

and

$$
g_{h_{k, v}, u}=g_{k,(u, H v)} w
$$

for some $\left.w \in G_{\sigma_{k}(u, H} v\right)$. We get the following commutative diagram:

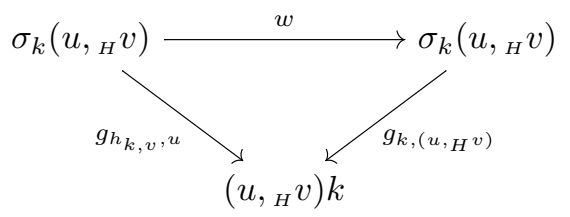

Using the commutativity of the above diagram and Lemma 25 we get

$$
\begin{aligned}
\mathfrak{L}_{V, \rho} \circ \mathfrak{L}_{U, \lambda}\left(k, f, f^{\prime}\right)= & \prod_{v \in[H \backslash V]} \mathfrak{L}_{U, \lambda}\left(h_{k, v}, f\left(\sigma_{k}(v)\right), f^{\prime}(v)\right) \rho^{-1}\left(h_{k, v}, k, \sigma_{k}(v), v\right) \\
= & \prod_{\substack{u \in[G \backslash U] \\
v \in[H \backslash V]}} \mathfrak{l}\left(g_{h_{k, v}, u}, f\left(\sigma_{k}(v)\right)\left(\sigma_{h_{k, v}}(u)\right), f^{\prime}(v)(u)\right) \\
& \lambda^{-1}\left(g_{h_{k, v}, u}, h_{k, v}, \sigma_{h_{k, v}}(u), u\right) \rho^{-1}\left(h_{k, v}, k, \sigma_{k}(v), v\right) \\
= & \prod_{\left(u,,_{H} v\right) \in\left[G \backslash\left(U \times_{H} V\right)\right]} \mathfrak{l}\left(g_{h_{k, v}, u}, f\left(\sigma_{h_{k, v}}(u),{ }_{H} \sigma_{k}(v)\right), f^{\prime}\left(u,{ }_{H} v\right)\right) \\
& (\lambda \times \rho)^{-1}\left(g_{h_{k, v}, u}, k,\left(\sigma_{h_{k, v}}(u),{ }_{H} \sigma_{k}(v)\right),\left(u,{ }_{H} v\right)\right) \\
= & \prod_{\left(u,{ }_{H} v\right) \in\left[G \backslash\left(U \times_{H} V\right)\right]} \mathfrak{l}\left(g_{k,\left(u,{ }_{H} v\right)} w, f\left(\sigma_{k}\left(u,{ }_{H} v\right)\right), f^{\prime}\left(u,{ }_{H} v\right)\right) \\
& (\lambda \times \rho)^{-1}\left(g_{k,\left(u,,_{H} v\right)} w, k,\left(\sigma_{h_{k, v}}(u),{ }_{H} \sigma_{k}(v)\right),\left(u,{ }_{H} v\right)\right) \\
= & \prod_{\left(u,{ }_{H} v\right) \in\left[G \backslash\left(U \times_{H} V\right)\right]} \mathfrak{l}\left(g_{k,\left(u,,_{H} v\right)}, f\left(\sigma_{k}\left(u,{ }_{H} v\right)\right), f^{\prime}\left(u,{ }_{H} v\right)\right) \\
& \left.(\lambda \times \rho)^{-1}\left(g_{k,(u, H} v\right), k,\left(\sigma_{h_{k, v}}(u),{ }_{H} \sigma_{k}(v)\right),\left(u,{ }_{H} v\right)\right) \\
= & \mathfrak{L}_{U \times{ }_{H} V}(X, \mathfrak{l}) . \quad \square
\end{aligned}
$$

Remark 27. The following example shows that the assumption that $V$ is left free seems to be necessary for Assertion 5. Suppose that $H=N \rtimes K$ is a semidirect product of a normal subgroup $N$ with $K$. Let $G$ be the group $K$, viewed as a subgroup of $H$. Let moreover $U$ be the set $H$, viewed as a $(G, H)$-biset by left and right multiplication, and 
let $V$ be the set $K$, acted on by $K$ on the right by multiplication, and by $H$ on the left by projection to $K=H / N$, followed by multiplication in $K$. Let moreover $\lambda$ and $\rho$ be equal to the trivial functor on $\widehat{U}$ and $\widehat{V}$, respectively.

Then $U_{\lambda} \circ_{H} V_{\rho}=U \times_{H} V$, as $\lambda$ and $\rho$ are both trivial. Moreover $U \times_{H} V=G \times_{H} K$ is equal to the identity $(K, K)$-biset (this makes sense since $G=K$ ), so $T_{U \times_{H} V, \lambda \times \rho}=$ $T_{\mathrm{id}_{K}, 1}$ is the identity functor, by Assertion 4 .

On the other hand $G \backslash U=K \backslash(N K) \cong N$, and $H \backslash V$ has cardinality 1 . So in the computation of the functor $\mathcal{L}_{U, 1}$ appearing in $T_{U, 1}(X, \mathfrak{l})$, we have a product of values of $\mathfrak{l}$, indexed by $N$. So the composition $T_{V, 1} \circ T_{U, 1}$ cannot act in general as the identity on $(X, \mathfrak{l})$, if $N$ is non trivial. Hence $T_{V, \lambda} \circ T_{U, \rho} \neq T_{U \times_{H} V, \lambda \times \rho}$ in this situation.

Remark 28. Let $G$ and $H$ be finite groups, and $U$ be a (finite) $(G, H)$-biset. Then one can check that the diagram

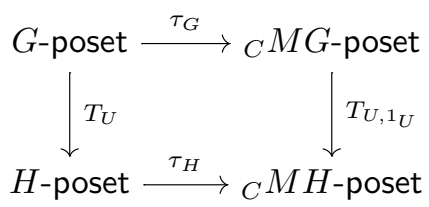

of categories and functors is commutative, up to isomorphism, where the functor $T_{U}$ on the left is the usual generalized tensor induction functor for $G$-posets.

Lemma 29. Let $G$ and $H$ be finite groups, and let $(U, \lambda)$ be a $C$-monomial $(G, H)$-biset. Then there exists a unique map

$$
\mathcal{T}_{U, \lambda}: B_{C}(G) \rightarrow B_{C}(H)
$$

such that $\mathcal{T}_{U, \lambda}\left(\Lambda_{(X, \mathfrak{l})}\right)=\Lambda_{T_{U, \lambda}(X, \mathfrak{l})}$ for any finite $C$-monomial $G$-poset $(X, \mathfrak{l})$.

Proof. We show that if $(X, \mathfrak{l})$ and $(Y, \mathfrak{m})$ are finite $C$-monomial $G$-posets such that if $\Lambda_{(X, \mathfrak{l})}=\Lambda_{(Y, \mathfrak{m})}$ in $B_{C}(G)$, then $\Lambda_{T_{U, \lambda}(X, \mathfrak{l})}=\Lambda_{T_{U, \lambda}(Y, \mathfrak{m})}$ in $B_{C}(H)$. So it's enough to show that $\chi\left(T_{U, \lambda}((X, \mathfrak{l}))^{K, \theta}\right)=\chi\left(T_{U, \lambda}((Y, \mathfrak{m}))^{K, \theta}\right)$, by Lemma 20 for any $(K, \theta)$ of $\operatorname{ch}(G)$.

Let $u \in[G \backslash U / K], k \in K$ and $t \in\left[K \cap G^{u} \backslash K\right]$ then there exist a unique $\sigma_{k}(u t) \in[G \backslash U]$ and some $g_{k, u t} \in G$ such that

$$
u t k=g_{k, u t} \sigma_{k}(u t)
$$

Also there exist some $c_{k, t} \in K \cap G^{u}$ and a unique $\tau_{k}(t) \in\left[K \cap G^{u} \backslash K\right]$ such that

$$
t k=c_{k, t} \tau_{k}(t)
$$

Since $c_{k, t} \in K \cap G^{u}$, there exists $\gamma_{k, t, u} \in G$ such that 


$$
u c_{k, t}=\gamma_{k, t, u} u
$$

Now

$$
u t k=u c_{k, t} \tau_{k}(t)=\gamma_{k, t, u} u \tau_{k}(t)=g_{k, u t} \sigma_{k}(u t)
$$

So $\sigma_{k}(u t)=u \tau_{k}(t)$ and there exists $w \in G_{\sigma_{k}(u t)}$ such that $g_{k, u t}=\gamma_{k, t, u} w$. We get the following commutative diagram:

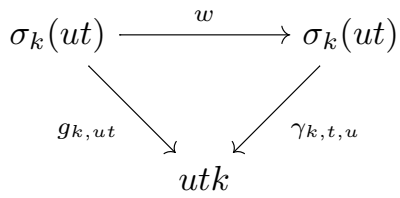

Now let $f \in t_{U, \lambda}(X, \mathfrak{l})^{K, \theta}$ and $k \in K$ such that $k f=f$. Note that since $f$ is $K$-fixed, we have

$$
f(u t k)=f(u t)=f(u)=\gamma_{k, t, u} f\left(u \tau_{k}(t)\right)=\gamma_{k, t, u} f(u)
$$

so $\gamma_{k, t, u} \in G_{f(u)}$. Hence

$$
f\left(\sigma_{k}(u t)\right)=f\left(u \tau_{k}(t)\right)=\gamma_{k, t, u} f(u t k)=\gamma_{k, t, u} f(u)=f(u) .
$$

Let $\gamma_{k, u}=\prod_{t \in\left[K \cap G^{u} \backslash K\right]} \gamma_{k, t, u}$ and $\phi_{u}(k)=\prod_{t \in\left[K \cap G^{u} \backslash K\right]} \lambda^{-1}\left(\gamma_{k, t, u}, k, \sigma_{k}(u t), u t\right)$. Then

$$
\begin{aligned}
& \mathfrak{L}(k, f, f)=\prod_{u \in[G \backslash U]} \mathfrak{l}\left(g_{k, u}, f\left(\sigma_{k}(u)\right), f(u)\right) \lambda^{-1}\left(g_{k, u}, k, \sigma_{k}(u), u\right) \\
& =\prod_{\substack{u \in[G \backslash U / K] \\
t \in\left[K \cap G^{u} \backslash K\right]}} \mathfrak{l}\left(g_{k, u t}, f\left(\sigma_{k}(u t)\right), f(u t)\right) \lambda^{-1}\left(g_{k, u t}, k, \sigma_{k}(u t), u t\right) \\
& =\prod_{\substack{u \in[G \backslash U / K] \\
t \in\left[K \cap G^{u} \backslash K\right]}} \mathfrak{l}\left(\gamma_{k, t, u} w, f\left(\sigma_{k}(u t)\right), f(u t)\right) \lambda^{-1}\left(\gamma_{k, t, u} w, k, \sigma_{k}(u t), u t\right) \\
& =\prod_{\substack{u \in[G \backslash U / K] \\
t \in\left[K \cap G^{u} \backslash K\right]}} \mathfrak{l}\left(\gamma_{k, t, u}, f\left(\sigma_{k}(u t)\right), f(u t)\right) \lambda^{-1}\left(\gamma_{k, t, u}, k, \sigma_{k}(u t), u t\right) \\
& =\prod_{u \in[G \backslash U / K]} \mathfrak{l}_{f(u)}\left(\gamma_{k, u}\right) \phi_{u}(k) \\
& =\theta(k) \text {. }
\end{aligned}
$$

Let $\Xi$ be the family of the sets $\xi=\left\{\xi_{u}\right\}_{u \in[G \backslash U / K]}$ where $\xi_{u}:{ }^{u} K \rightarrow C$ is a character such that $\operatorname{res}_{u}^{G_{f(u)}}\left(\mathfrak{l}_{f(u)}\right)=\xi_{u}$ and 


$$
\theta(k)=\prod_{u \in[G \backslash U / K]} \xi_{u}\left(\gamma_{k, u}\right) \phi_{u}(k)
$$

for all $k \in K$ and $u \in[G \backslash U / K]$.

We claim that

$$
T_{U, \lambda}(X, \mathfrak{l})^{K, \theta}=\bigsqcup_{\xi \in \Xi} \prod_{u \in[G \backslash U / K]}(X, \mathfrak{l})^{u} K, \xi_{u} .
$$

Let $f \in T_{U, \lambda}(X, \mathfrak{l})^{K, \theta}$, then $f(g u k)=g f(u)$ for all $g \in G, u \in U$, and $k \in K$. So to determine $f$, it's enough to know $f(u)$ for $u \in[G \backslash U / K]$. Let $f(u)=x_{u}$. Then

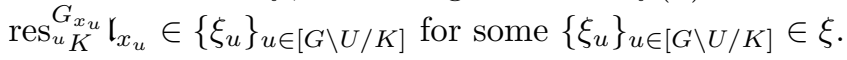

Conversely, let us choose $x_{u} \in X$ for any $u \in[G \backslash U / K]$. Let $v \in V$ then $v=g u k$ for some $g \in G$, for some $k \in K$ and a unique $u \in[G \backslash U / K]$. We set $f(v)=g x_{u}$. Now $f$ is well defined if and only if $g x_{u}=x_{u}$ whenever $g \in{ }^{u} K$ or equivalently $x_{u} \in X^{u} K$. We want that $f \in T_{U, \lambda}(X, \mathfrak{l})^{K, \theta}$. If $x_{u} \in(X, \mathfrak{l})^{u} K, \xi_{u}$ then $\operatorname{res}_{u_{K}}^{G_{x_{u}}}\left(\mathfrak{l}_{x_{u}}\right)=\xi_{u}$ and

$$
\theta(k)=\prod_{u \in[G \backslash U / K]} \xi_{u}\left(\gamma_{k, u}\right) \phi_{u}(k)
$$

So $f \in T_{U, \lambda}(X, \mathfrak{l})^{K, \theta}$.

Now using [[2], Lemma 11.2.9] we get

$$
\chi\left(T_{U, \lambda}(X, \mathfrak{l})^{K, \theta}\right)=\sum_{\xi \in \Xi} \prod_{u \in[G \backslash U / K]} \chi\left((X, \mathfrak{l})^{u} K, \xi_{u}\right) .
$$

Thus, if $\Lambda_{(X, \mathfrak{l})}=\Lambda_{(Y, \mathfrak{m})}$ then $\Lambda_{T_{U, \lambda}(X, \mathfrak{l})}=\Lambda_{T_{U, \lambda}(Y, \mathfrak{m})}$. So we can define a map

$$
\mathcal{T}_{U, \lambda}: B_{C}(G) \rightarrow B_{C}(H)
$$

such that $\mathcal{T}_{U, \lambda}(a)=\Lambda_{T_{U, \lambda}(X, \mathfrak{l})}$ where $(X, \mathfrak{l})$ is a $C$-monomial $G$-poset such that $a=\Lambda_{(X, \mathfrak{l})}$, as in Corollary 11.

Proposition 30. Let $G$ and $H$ be finite groups, and let $(U, \lambda)$ be a $C$-monomial $(G, H)$-biset.

1. $\mathcal{T}_{U, \lambda}\left(\left[G, 1_{G}\right]_{G}\right)=\left[H, 1_{H}\right]_{H}$.

2. $\mathcal{T}_{U, \lambda}(a b)=\mathcal{T}_{U, \lambda}(a) \mathcal{T}_{U, \lambda}(b)$, for any $a, b \in B_{C}(G)$.

In particular, the restriction of $\mathcal{T}_{U, \lambda}$ to $B_{C}(G)^{\times}$is a group homomorphism

$$
\mathcal{T}_{U, \lambda}^{\times}: B_{C}(G)^{\times} \rightarrow B_{C}(H)^{\times}
$$


Proof. 1. Consider the $C$-monomial $G$-poset $(\bullet, 1)$ then clearly $\Lambda_{(\bullet, 1)}=\left[H, 1_{H}\right]_{H}$. So using the first assertion of Proposition 26 we get

$$
\mathcal{T}_{U, \lambda}\left([G, 1]_{G}\right)=\Lambda_{T_{U, \lambda}(\bullet, 1)}=\Lambda_{(\bullet, 1)}=\left[H, 1_{H}\right]_{H}
$$

2. Let $a, b \in B_{C}(G)$ then by Corollary 11 there exist $C$-monomial $G$-posets $(X, \mathfrak{l})$ and $(Y, \mathfrak{m})$ such that $\Lambda_{(X, \mathfrak{l})}=a$ and $\Lambda_{(Y, \mathfrak{m})}=b$. Then

$$
\begin{aligned}
\mathcal{T}_{U, \lambda}(a b) & =\mathcal{T}_{U, \lambda}\left(\Lambda_{(X, \mathfrak{l})} \Lambda_{(Y, \mathfrak{m})}\right)=\mathcal{T}_{U, \lambda}\left(\Lambda_{X \times Y, \mathfrak{l} \times \mathfrak{m}}\right)=\Lambda_{T_{U, \lambda}(X \times Y, \mathfrak{l} \times \mathfrak{m})} \\
& =\Lambda_{T_{U, \lambda}(X, \mathfrak{l})} \Lambda_{T_{U, \lambda}(Y, \mathfrak{m})}=\mathcal{T}_{U, \lambda}(a) \mathcal{T}_{U, \lambda}(b) \quad \square
\end{aligned}
$$

Proposition 31. Let $G, H$, and $K$ be finite groups.

1. Let $\operatorname{id}_{G}$ stand for the identity $(G, G)$-biset. Then $\mathcal{T}_{\mathrm{id}_{G}, 1_{G}}$ is the identity map of $B_{C}(G)$.

2. Let $(U, \lambda)$ and $\left(U^{\prime}, \lambda^{\prime}\right)$ be $C$-monomial $(G, H)$-bisets. Then for any $a \in B_{C}(G)$

$$
\mathcal{T}_{U \sqcup U^{\prime}, \lambda \sqcup \lambda^{\prime}}(a)=\mathcal{T}_{U, \lambda}(a) \mathcal{T}_{U^{\prime}, \lambda^{\prime}}(a)
$$

3. Let $(U, \lambda)$ be a C-monomial $(G, H)$-biset and let $(V, \rho)$ be a monomial left free $(H, K)$-biset then

$$
\mathcal{T}_{V, \rho} \circ \mathcal{T}_{U, \lambda}=\mathcal{T}_{U \times_{H} V, \lambda \times \rho}
$$

Proof. Let $a \in B_{C}(G)$ then by Corollary 11 there exists a $C$-monomial $G$-poset $(X, \mathfrak{l})$ such that $a=\Lambda_{(X, \mathfrak{l})}$.

1. Using the third assertion of Proposition 26, we get

$$
\mathcal{T}_{\operatorname{id}_{G}, 1_{G}}(a)=\mathcal{T}_{\operatorname{id}_{G}, 1_{G}}\left(\Lambda_{(X, \mathfrak{l})}\right)=\Lambda_{T_{\operatorname{id}_{G}, 1_{G}}(X, \mathfrak{l})}=\Lambda_{(X, \mathfrak{l})}=a
$$

2. Using the second assertion of Proposition 26, we get

$$
\begin{aligned}
\mathcal{T}_{U \sqcup U^{\prime}, \lambda \sqcup \lambda^{\prime}}(a) & =\mathcal{T}_{U \sqcup U^{\prime}, \lambda \sqcup \lambda^{\prime}}\left(\Lambda_{(X, \mathfrak{l})}\right)=\Lambda_{T_{U \sqcup U^{\prime}, \lambda \sqcup \lambda^{\prime}}(X, \mathfrak{l})} \\
& =\Lambda_{T_{U, \lambda}(X, \mathfrak{l}) \times T_{U^{\prime}, \lambda^{\prime}}(X, \mathfrak{l})}=\Lambda_{T_{U, \lambda}(X, \mathfrak{l})} \Lambda_{T_{U^{\prime}, \lambda^{\prime}}(X, \mathfrak{l})} \\
& =\mathcal{T}_{U, \lambda}\left(\Lambda_{(X, \mathfrak{l})}\right) \mathcal{T}_{U^{\prime}, \lambda^{\prime}}\left(\Lambda_{(X, \mathfrak{l})}\right)=\mathcal{T}_{U, \lambda}(a) \mathcal{T}_{U^{\prime}, \lambda^{\prime}}(a) .
\end{aligned}
$$

3. Using the fourth assertion of Proposition 26, we get

$$
\begin{aligned}
\mathcal{T}_{V, \rho} \circ \mathcal{T}_{U, \lambda}(a) & =\mathcal{T}_{V, \rho} \circ \mathcal{T}_{U, \lambda}\left(\Lambda_{(X, \mathfrak{l})}\right)=\Lambda_{T_{V, \rho} \circ T_{U, \lambda}(X, \mathfrak{l})} \\
& =\Lambda_{T_{U \times_{H} V, \lambda \times \rho}(X, \mathfrak{l})}=\mathcal{T}_{U \times_{H} V, \lambda \times \rho}\left(\Lambda_{(X, \mathfrak{l})}\right)=\mathcal{T}_{U \times_{H} V, \lambda \times \rho}(a) .
\end{aligned}
$$


Corollary 32. Let $G$ and $H$ be finite groups. The map $(U, \lambda) \mapsto \mathcal{T}_{U, \lambda}^{\times}$of Proposition 30 extends to a bilinear map

$$
B_{C}(G, H) \times B_{C}(G)^{\times} \rightarrow B_{C}(H)^{\times}
$$

Proof. This follows from Assertion 2 of Proposition 26 and Assertion 2 of Proposition 31, and from the fact that the map $\mathcal{T}_{(U, \lambda)}^{\times}$depends only on the isomorphism class of $(U, \lambda)$.

Remark 33. It follows from Remark 28 that if $U$ is a finite $(G, H)$-biset, the square

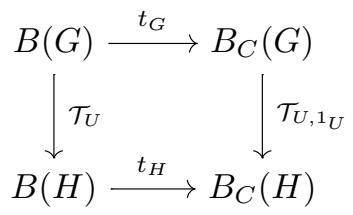

of groups and multiplicative maps, is commutative, where $\mathcal{T}_{U}$ on the left is the usual generalized tensor induction map for Burnside rings, and the horizontal maps $t_{G}$ and $t_{H}$ are the ring homomorphisms induced by the functors $\tau_{G}$ and $\tau_{H}$.

\section{Acknowledgments}

The second author was granted the Fellowship Program for Abroad Studies BIDEP2214-A by the Scientific and Technological Research Council of Turkey (Tübitak). The second author also wishes to thank LAMFA for their hospitality during the visit.

\section{References}

[1] L. Barker, Fibred permutation sets and the idempotents and units of monomial Burnside rings, J. Algebra 281 (2004) 535-566.

[2] S. Bouc, Biset Functors for Finite Groups, Lecture Notes in Math., vol. 1990, Springer, Berlin, 2010.

[3] S. Bouc, Burnside Rings, Handb. Algebr., vol. 2, 2000, pp. 739-804.

[4] R. Carman, Unit groups of representation rings and their ghost rings as inflation functors, J. Algebra 498 (2018) 263-293.

[5] A.W.M. Dress, The ring of monomial representations, I. Structure theory, J. Algebra 18 (1971) $153-157$.

[6] D. Quillen, Higher Algebraic K-Theory, Lecture Notes in Math., vol. 341, Springer, Berlin, 1973, pp. $85-147$.

[7] J. Thévenaz, Permutation representation arising from simplicial complexes, J. Combin. Theory Ser. A 46 (1987) 122-155. 